To appear in the Astronomical Journal

\title{
The Sizes of the Nearest Young Stars
}

\author{
Kyle McCarthy ${ }^{1,2}$ and Russel J. White ${ }^{1}$ \\ ${ }^{1}$ Department of Physics and Astronomy, Georgia State University, Atlanta, GA 30303-4106 \\ ${ }^{2}$ Department of Physics and Astronomy, University of Kentucky, Lexington, KY \\ 40508-0055 \\ kyle.mccarthy@uky.edu
}

\begin{abstract}
We present moderate resolution $(\mathrm{R} \sim 3575)$ optical spectra of 19 known or suspected members of the $\mathrm{AB}$ Doradus and $\beta$ Pictoris Moving Groups, obtained with the DeVeny Spectrograph on the 72-inch Perkins telescope at Lowell Observatory. For 4 of 5 recently proposed members, signatures of youth such as Li I $6708 \AA$ absorption and $\mathrm{H} \alpha$ emission further strengthen the case for youth and membership. The lack of detected lithium in the proposed $\beta$ Pic member TYC 2211-1309-1 implies that it is older than all other K-type members, and weakens the case for membership. Effective temperatures are determined via line ratio analyses for the $11 \mathrm{~F}, \mathrm{G}$ and early $\mathrm{K}$ stars observed, and via spectral comparisons for the 8 late- $\mathrm{K}$ and $\mathrm{M}$ stars observed. We assemble updated candidate membership lists for these Moving Groups that account for known binarity. Currently the AB Dor moving group contains 127 proposed members and the /beta Pic moving group holds 77 proposed members. We then use temperature, luminosity, and distance estimates to predict angular diameters for these stars; the motivation is to identify stars that can be spatially resolved with long-baseline optical/infrared interferometers in order to improve age estimates for these Groups and to constrain evolutionary models at young ages. Considering the portion of the sky accessible to northern hemisphere facilities (DEC > -30), 6 stars have diameters large enough to be spatially resolved $(\theta>0.4$ mas) with the CHARA Array, which currently has the world's longest baseline of 331-m; this subsample includes the low mass M2.5 member of AB Dor, GJ 393, which is likely to still be pre-main sequence. For southern hemisphere facilities $(\mathrm{DEC}<+30)$, 18 stars have diameters larger than this limiting size, including the low mass debris disk star AU Mic (0.72 mas). However, the longest baselines of southern
\end{abstract}


hemisphere interferometers (160-m) are only able to resolve the largest of these, the B6 star $\alpha$ Gru (1.17 mas); proposed long-baseline stations may alleviate the current limitations.

Subject headings: Techniques: interferometric, Stars: binaries: general, Stars: fundamental parameters, Stars: pre-main sequence

\section{Introduction}

Stellar evolutionary models are an essential tool in the study of star and planet formation since, in most cases, they provide the only means of estimating fundamental stellar properties like mass and age. Despite decades of progress with these models, there remain considerable uncertainties in the requisite input physics (Baraffe et al. 2002; Young \& Arnett 2005). For main-sequence stars, input parameters such as mixing length or abundances can be tuned to predict properties consistent with observations; this is in large part because of the wealth of precise dynamical masses and stellar radii that high resolution techniques such as interferometry have provided (e.g. Michelson \& Pease 1921; Hanbury Brown et al. 1974; Labeyrie 1975). Unfortunately, evolutionary models are considerably more uncertain at premain sequence ages. While this is partially due to the additional complicating properties of young stars like rapid rotation and star spots, it is also a consequence of most star forming regions being too distant $(>100 \mathrm{pc}$ ) to permit detailed interferometric measurements of their members. As a consequence, mass estimates for young stars vary by as much as 50\%-200\% and age estimates vary by up to a factor of 10, depending upon the adopted evolutionary model (e.g. Hillenbrand \& White 2004; Mathieu et al. 2007).

Fortunately, two advances in observational astronomy over the last decade are enabling new fundamental measurements of young stars. The first is the discovery of many young $(\lesssim 100 \mathrm{Myr})$ stars within close proximity of the Sun (e.g. Zuckerman \& Song 2004a; López-Santiago et al. 2006; Torres et al. 2008; Lépine \& Simon 2009). The majority of these young stars are members of small, slowly dispersing $\mathrm{T}$ Associations, now commonly referred to as Moving Groups. As cataloged in the recent review article by Torres et al. (2008), there are now 9 well defined Moving Groups, all of which have ages less than $\sim 100 \mathrm{Myr}$ and 4 of which have central distances within 60 pc of the Sun. Because of their close proximity, many of these stars also have well determined distances from the Hipparcos parallax mission (Perryman et al. 2009), which enable much more precise stellar luminosity and size estimates.

An important complement to these discoveries are rapid advances in long-baseline inter- 
ferometry. Facilities now operate at infrared and even optical wavelengths and can achieve spatial resolutions better than 1 milliarcsecond (mas). For comparison, at a distance of 10 pc, the sun would have and angular diameter of 0.93 mas. Currently, the longest baseline optical/infrared interferometer is Georgia State University's CHARA Array, located on Mt. Wilson in California (ten Brummelaar et al. 2005). With a long baseline of 331-m, the Array yields a minimum angular resolution below 1 mas in the infrared (e.g. Boyajian et al. 2008; Baines et al. 2010); the smallest published angular diameter measured by CHARA is that of HD 189733 with an angular size of $0^{\prime \prime} .377 \pm 0.024$ mas in the H-band (Baines et al. 2007). This resolution limit will continue to improve as instruments that operate optical wavelengths are utilized (e.g. Mourard et al. 2009). Other operational long-baseline interferometers include the Navy Prototype Optical Interferometer in Arizona (Hummel et al. 2003), the Very Large Telescope Interferometer (Petrov et al. 2007) located on Cerro Paranal, Chile, and the Sydney University Stellar Interferometer (Davis et al. 1999) in New South Wales, Australia. Although these interferometers have thus far utilized baselines less than 160-m, the proposed extension to longer baselines and observations at optical wavelengths suggest they will achieve similar spatial resolutions to the CHARA Array in the near future.

The primary purpose of this article is to investigate which, if any, of the nearest young stars are large enough and bright enough to be spatially resolved by the current generation of optical/infrared interferometers. We also comment on the types of improvements that would dramatically increase the number of young stars that could be spatially resolved.

\section{The Nearest Young Stars}

In this analysis, we focus on the two nearest Moving Groups, AB Doradus and $\beta$ Pictoris. Torres et al. (2008) list mean distances of 34 and 31 pc with dispersions of 26 and 31 pc for these groups, respectively. These distances are $\sim 30 \%$ closer than the next closest Moving Groups, Tucana/Horologium and TW Hydrae, both at an average distance of 48 pc. In addition, unlike the Tucana/Horologium and TW Hydrae Moving Groups, both AB Doradus and $\beta$ Pictoris have many members in the northern hemisphere and thus are accessible to the CHARA Array.

$A B$ Doradus ( $A B$ Dor): Age estimates for the AB Dor Moving Group range from being comparable to the youngest known open clusters (30-50 Myr; Zuckerman et al. 2004b; Close et al. 2005) to being coeval with the Pleiades (100-125 Myr; Ortega et al. 2007; Luhman et al. 2005). Based on an analysis of lithium abundances of known members, da Silva et al. (2009) favor an intermediate age of 70 Myr (see also Janson et al. 2007). 
In Table 1 we provide a current list of 127 potential members of this Moving Group, along with distance estimates, Johnson $V$-band magnitudes, spectral types, $K_{2 M A S S}$ magnitudes, assigned temperatures and multiplicity status. The majority of these members come from the compilation paper of Torres et al. (2008), who create a membership list based upon the previous studies of Covino et al. (1997); Zuckerman et al. (2004b); Zickgraf et al. (2005); López-Santiago et al. (2006). Torres et al. (2008) cite a membership probability for each star based on evolutionary and kinematic criteria, as described in Torres et al. (2006). The membership probabilities span from $60 \%$ to $100 \%$. To this list we added the 6 proposed members from Schlieder et al. (2010), who identify members based on trigonometric distances, X-ray emission, $\mathrm{H} \alpha$ emission, and radial velocity measurements. Finally, we add 3 candidate members from da Silva et al. (2009) 1 who use the same criteria as Torres et al. (2008) to identify candidate members, and 7 candidates from Zuckerman et al. (2011) who use galactic space motions, locations on a color-magnitude diagram, lithium abundances, and X-ray luminosities to identify candidate members; the 7 stars identified in Zuckerman et al. (2011) are all A- or late B-type stars.

Of these 127 stars, 54 have distances determined from Hipparcos parallax measurements (van Leeuwen 2007, marked with an H in Table 1). Another 40 stars have distances estimated kinematically by Torres et al. (2008) or Schlieder et al. (2010). Any companions (defined below) to these stars are assumed to be at the same distance. Only 3 of the 127 stars have no distance estimates (HW Cet, TYC 0091-0082-1, RX J0928.5-7815). For these stars, we estimate their distances by assuming they have the same radii (calculated below) as stars of the same spectral type, and then determine the distances that would yield a luminosity consistent with their observed $V$ magnitudes; these calculated distances are marked in the Tables.

The Johnson $V$-band magnitudes are calculated in the majority of cases (97 stars) from $V_{T}$ magnitudes in the Tycho-2 Catalog (Høg et al. 2000), using the prescription of Bessell (2000), which relies on knowing the $B_{T}$ and $V_{T}$ magnitudes. For the 4 stars that only have a $V_{T}$ magnitude we estimate their $\left(B_{T}-V_{T}\right)$ color using their spectral type and the color relations of Hartigan et al. (1994). In the absence of any Tycho- 2 magnitudes, $V$-band magnitudes are assembled from measurements in the literature, when available. Stars with literature $V$ magnitudes include AB Dor BaBb (Collier-Cameron \& Foing 1997), GSC 88940426 (Craig et al. 1997), GSC 8544-1037, CD-45 14955 (Torres et al. 2006, 2008), and $\alpha$ Gru and $\delta$ Scl (Zuckerman et al. 2011). In total 107 of the 127 potential members have $V$-band measurements. We note that the $V$ magnitudes of 14 stars represent the combined light of

\footnotetext{
${ }^{1}$ The newly proposed AB Dor member BD+09 412 by da Silva et al. (2009) is also HW Cet; the SIMBAD database does not currently recognize these 2 stars as being the same star.
} 
2 or more stars (values listed in brackets in the Tables).

Of the assembled 2MASS $K$-band magnitudes, 17 are of 2 or more stars (value listed in brackets). Fewer pairs have resolved 2MASS measurements than $V$-band measurements because of the higher resolution of the Tycho instrument $\left(\sim 00^{\prime \prime} 8\right.$; Høg et al. 2000) compared to the 2MASS survey ( $\sim 3$ "; Skrutskie et al. 2006). Although the candidate brown dwarf companion to CD-35 2722 A (Wahhaj et al. 2011) is spatially unresolved at $V$ and $K$, we do not mark the measurement as "combined", given the very low flux ratio measured for this pair at infrared wavelengths.

The assembled spectral types are as listed in Torres et al. (2008) or the discovery paper for more recent additions. These spectral types are used to estimate temperatures following the temperature-spectral type scale in Hartigan et al. (1994) for F, G, K, and M stars and Kenyon \& Hartmann (1995) for B and A stars. There are 4 stars without a spectral type (BD+23 296 B, HW Cet, AK Pic B, and CD-49 2843 B), but fortunately all have Tycho-2 $B_{T}$ and $V_{T}$ from which spectral types and temperatures can be estimated using the above color relations. These estimated spectral types are marked with a colon in Tables 1 and 2 .

In the last column of Table 1 we describe the multiplicity status of each star (single, binary, triple, quadruple), if known 3 . Stars are only listed as single if they have been included in high spatial resolution imaging surveys (Kasper et al. 2007; Biller et al. 2010; Nielson \& Close 2010; Evans et al. 2011) and no companion was identified. For multiples, projected separations are given if the pair has been spatially resolved. The separations for pairs closer than $\sim 10^{\prime \prime} 0$ are as assembled in Torres et al. (2008), while wider pairs are calculated from the stars' 2MASS positions.

We classify 26 systems as multiple within the AB Dor Moving Group by assuming that all systems with projected separations less than 75".0 are physically associated. This separation limit is set in order to classify the $\beta$ Pic triples 51 Eri \& GJ 3305 AB and HR 7012 AB \& CD-64 1208 B as physical systems. Although the separation limit is somewhat subjective, the projected separations in these systems correspond to distances of 1979 AU and 2163

\footnotetext{
${ }^{2}$ This publication makes use of data products from the Two Micron All Sky Survey, which is a joint project of the University of Massachusetts and the Infrared Processing and Analysis Center/California Institute of Technology, funded by the NASA and the NSF.

3 Torres et al. (2008) provides a useful Table summarizing known binaries in AB Dor, to which we note the following corrections. UY Pic AB is listed with a separation of $10^{\prime \prime} 3$ in their Table 14, but its separation is $18^{\prime \prime} .3$ in the 2MASS database. The $16^{\prime \prime} 2$ companion listed to HD 45270 can not be confirmed; we do not include this companion in our list. We assume that the spectroscopic companion to PX Vir reported in Torres et al. (2008) is the same $\sim 00^{\prime \prime} 4$ companion spatially resolved by Evans et al. (2011).
} 
AU, which is consistent with many known physically bound binaries (Raghavan et al. 2010), while all wider pairs have projected separations greater than 12,000 AU. We nevertheless note that CD-60 1425 \& GSC 8894-0426 (27.2 arcmin), BD+08 4561 \& TYC 1090-543-1 (4.1 arcmin) and AK Pic AB \& CD-61 1439 (13.4 arcmin) are all spatially close on the sky.

$\beta$ Pictoris ( $\beta$ Pic): The age of this cluster is more accurately determined than that of AB Dor, primarily because this Moving Group is younger and thus more distinct from the zero age main sequence. Studies of its ensemble population suggest ages that range from 10 to 21 Myr (Zuckerman et al. 2001; Feigelson et al. 2006; Mentuch et al. 2008; da Silva et al. 2009, but, cf. Yee \& Jensen 2010, Lépine \& Simon 2009, MacDonald \& Mullan 2010).

In Table 2 we provide a current list of 77 potential members of this Moving Group along with distance estimates, Johnson $V$-band magnitudes, $K_{2 M A S S}$ magnitudes, spectral types, assigned temperatures and multiplicity status. The majority of these members come from the compilation of Torres et al. (2008)4, who created a membership list based upon the previous studies of Moór et al. (2006) and Zuckerman \& Song (2004a). More recently, Lépine \& Simon et al. (2009), Schlieder et al. (2010) and Kiss et al. (2011) have identified another 13 candidate members which we include in our sample. Lépine \& Simon et al. (2009) and Schlieder et al. (2010) used methods described above for identifying members, while Kiss et al. (2011) relied on 3 age diagnostics: color-magnitude diagrams, X-ray emission and lithium abundance.

Of these 77 potential members, 28 stars have distances determined from Hipparcos parallax measurements (marked with an $\mathrm{H}$ in Table 2), and 27 have distances determined kinematically by Torres et al. (2008) or Lépine \& Simon (2009) or Kiss et al. (2011) or Schlieder et al. (2010). Companion stars are again assumed to have the same distance as the primary star (marked with an asterisk). Johnson $V$-band magnitudes are estimated in the majority of cases (47 stars) from the Tycho- 2 photometry, as described above. In the absence of Tycho-2 magnitudes, $V$-band magnitudes are assembled from the literature for several stars including AG Tri A, AG Tri B, GJ 3305 AB, V824 Ara C, GSC 8350-1924 AB, CD-51 11312 B, GSC 7396-0759, CD-64 1208 AB, 1 RXS J195602.8-320720, 1RXS J200136.9-331307, AT Mic A, AT Mic B, WW PsA, TX PsA (Torres et al. 2006), and V343 Nor B (Song et al. 2003). Fifteen stars do not have $V$-band measurements.

Eleven of the $V$-band measurements and 15 of the $K_{2 M A S S}$ measurements represent light from 2 or more stars, and are marked with brackets in Table 2; photometry for the 2 stars that harbor candidate brown dwarfs, PZ Tel (Biller et al. 2010) and $\eta$ Tel (Lowrance et al. 2000) are not marked because of their very low flux ratios. Temperatures are estimated for

\footnotetext{
${ }^{4}$ Torres et al. (2008) mislabel the $\beta$ Pic star HD14082 as HD 14062 in their Table 3.
} 
all stars using the assembled spectral types or Tycho color (for BD+17 232 B and HR 6749 B) using the same prescription described above.

There are 23 known multiple star systems within the $\beta$ Pic Moving Group using the same separation limit adopted above $\left(<75^{\prime \prime}\right)$. Although Torres et al. (2008) list BD+05 378 (HD 12545) as a spectroscopic binary, as reported by Song et al. (2003), more recent radial velocity observations show that it is not (Bailey et al. 2011). Although not classified as multiples, V4046 Srg AB \& GSC 7396-0759 (2.8 arcmin), $\eta$ Tel AB \& $\eta$ Tel C (6.9 arcmin), AT Mic AB \& AU Mic (77.9 arcmin), and HD 199143 \& AZ Cap (5.4 arcmin) are spatially close on the sky.

\section{Observations and Data Reduction}

Constraining evolutionary models depends critically upon having accurately determined stellar properties, such as temperature and luminosity, for comparisons with model predictions. This in part motivated the acquisition of optical spectra of Moving Group members. In particular, we obtained moderate resolution optical spectra of 19 stars in the $\beta$ Pic and AB Dor Moving Groups. These stars are listed in Table 3. Thirteen of these stars are well established members (probability > $95 \%$; Torres et al. 2008), one star has a more questionable membership (HD 15115; 60\% probability), and 5 stars are only recently identified candidate members from Lépine \& Simon (2009, TYC 1186-706-1, TYC 2211-1309-1, TYC 7443-1102-1, 1RXS J19506.8-3320720) and Schlieder et al. (2010, TYC 1752-63-1). In addition to these known or candidate young stars, we also obtained spectra of many stars with well known spectral types and/or effective temperatures from Kirkpatrick et al. (1991); Grav et al. (2001); Valenti \& Fischer (2005). All observations were obtained with the DeVeny spectrograph on the Perkins 72-inch Telescope at the Lowell Observatory during 3 observing runs: 2009 Feb 5, 2009 May 7, and 2009 Aug 1 - 3. On each night, we also obtained sets of bias images, dome-illuminated flat field spectra, and Neon-Argon lamp spectra for calibration purposes.

The spectrograph was used in combination with a $1200 \mathrm{~g} / \mathrm{mm}$ grating (blazed at $5000 \AA$ ). The spectra were projected onto a $2048 \times 515 \mathrm{CCD}$, with $1.0 \mathrm{arcsec} /$ pixel plate scale perpendicular to the dispersion. The resulting spectra had an average resolving power of 3575 , however the resolving power ranged from $\sim 3425$ at the edges to $\sim 3685$ at the center due to field curvature. These values were determined by measuring the widths of emission line features in the spectra of Neon-Argon lamps. The wavelength coverage was slightly different for these runs. In February and May, the wavelength range was $6250-7500 \AA$, while in August the wavelength range was shifted to 5950-7200 $\AA$ to avoid the fringing that becomes 
problematic ( $>$ few percent) longward of $\sim 7000 \AA$. No extinction corrections were applied to the spectra of these relatively close stars.

The images were reduced and the spectra were extracted using tools within the IRAF5 software package. An average bias frame was subtracted from each flat field image, and these were then median combined to generate a normalized master flat for each night. All stellar spectra were likewise bias subtracted and then divided by the master flats. The extracted spectra were background subtracted (using a median fit) and the continuum of these extracted spectra were fit with a 7 th order polynomial to produce normalized spectra. The Neon-Argon spectra were extracted identically and were used to assign an approximate wavelength solution for each night.

The observational epoch for each star is listed in Table 3 along with signal-to-noise ratio estimates for each spectrum. The signal-to-noise values were determined from the gaincorrected intensities in the center portions of the spectra. The February run is not listed for any of the stars as only spectral standards were observed during this (poor weather) run.

\section{Spectroscopically Inferred Properties}

For the 19 spectroscopically observed stars, measurements of the equivalent widths (EWs) of Li I $6708 \AA$ and $\mathrm{H} \alpha$ are presented in Section 4.1, and in Section 4.2 these measurements are used to further assess the membership status of recently proposed members; none of these proposed members have been previously observed at optical wavelengths. In Sections 4.3 and 4.4 we describe our methods for determining the effective temperatures of these stars.

\subsection{Equivalent Widths of $\mathrm{Li}[\mathrm{I}]$ and $\mathrm{H} \alpha$}

Two common diagnostics of stellar youth are the atmospheric abundance of lithium and the amount of chromospheric activity. Of these two diagnostics, lithium is the least ambiguous because of its rapid depletion with age, which is largely attributed to proton capture (e.g. Bodenheimer 1965). The strength of the $\mathrm{H} \alpha$ emission line is perhaps the most common tracer of chromospheric activity, but the persistence of $\mathrm{H} \alpha$ emission, especially for

\footnotetext{
${ }^{5}$ IRAF is distributed by the National Optical Astronomy Observatories, which are operated by the Association of Universities for Research in Astronomy, Inc., under cooperative agreement with the National Science Foundation.
} 
the lowest mass stars, makes it a much poorer diagnostic of age (West et al. 2008).

Figure 1 displays portions of DeVeny spectra near the $\mathrm{H} \alpha$ and lithium $6708 \AA$ features. The EWs of LiI $6708 \AA$ were determined by fitting a Gaussian profile to the absorption feature, using tools within IRAF. Because of the moderate resolution of these observations, the neighboring FeI line at $6708 \AA$ could not be removed from these measurements; the values may therefore be biased to larger EWs by $0.01-0.02 \AA$. To measure the EW of the $\mathrm{H} \alpha$ emission/absorption feature, a Gaussian profile was fit to this line after the deblending removal of the 5 nearby spectral features (telluric $\mathrm{H}_{2} \mathrm{O}$ at $6552 \AA$, $\mathrm{Si}[\mathrm{I}]$ at $6555 \AA, \mathrm{Fe}[\mathrm{I}]$ at $6569 \AA$, $\mathrm{Ca}[\mathrm{I}]$ at $6572 \AA$, and $\mathrm{Fe}[\mathrm{I}]$ at $6574 \AA$ ). A negative sign indicates emission, following convention. The resulting measurements are listed in Table 3 . The largest source of uncertainty in these measurements is the determination of the continuum level, a somewhat subjective process. Therefore we assign uncertainties based on the EW variations over a range of reasonable continuum levels. The EW uncertainties are on average $0.02 \AA$ for the lithium measurements and $10 \%$ for the $\mathrm{H} \alpha$ measurements.

The measured EW values are listed in Table 3. Of the 19 stars observed, 11 exhibit H $\alpha$ in emission and 15 have detectable lithium absorption. For the 4 stars without detectable lithium, detection upper limits are set by the continuum variations, and in most cases are 0.04 - 0.05 A. The one exception is 1RXS J19506.8-3320720, which has a low SNR spectrum and a much coarser lithium EW detection limit of $\sim 0.5 \AA$. As a consistency check, we compare our Li $6708 \AA$ measurements to previous measurements for the 13 stars with previously reported values. With 1 exception, the values are consistent to within $0.03 \AA$ with the values reported by Mentuch et al. (2008, 3 stars), Weise et al. (2010, 3 stars), López-Santiago et al. (2006, 2 stars), and Torres et al. (2006, 2 stars). The exception is LO Peg, for which we measure an EW of $0.150 \pm 0.02 \AA$ in a very high SNR spectrum, while López-Santiago et al. (2006) and da Silva et al. (2009) measure larger EW values of $0.233 \AA$ and $0.215 \AA$, respectively.

\subsection{Membership Confirmations}

Four stars observed here were recently proposed by Lépine \& Simon (2009) to be members of the $\beta$ Pic Moving Group (TYC 1186-706-1, TYC 2211-1309-1, TYC 7443-1102-1 and 1RXS J19506.8-3320720). All four exhibit H $\alpha$ emission, and 2 stars (TYC 1186-706-1, TYC 7443-1102-1) show clear Li I $6708 \AA$ absorption, which confirms youth and greatly strengthens the case for association with this Moving Group. Unfortunately the low signal-to-noise ratio spectrum of 1RXS J19506.8-3320720 prevented us from determining useful limits on the amount of lithium in its spectrum. Lithium is not detected in the spectrum of TYC 2211-1309-1 (EW < $0.04 \AA)$, which weakens the case for youth and association. This star's 
K7 spectral type (determined below) is many hundreds of Kelvin hotter than the temperature of stars predicted to deplete their lithium fastest (e.g. Baraffe et al. 1998; Yee \& Jensen 2010). For example, the spectral type M4 $\beta$ Pic members AT Mic A and AT Mic B exhibit no lithium in their spectra, but all high probability K7 members have strong Li I absorption (e.g. CP-72 2713: $0.44 \AA$; CD-31 16041: $0.49 \AA$; da Silva et al. 2009). While this suggests TYC 2211-1309-1 is likely older than known K-type members, it does not necessarily exclude it from the Moving Group; there could be a age spread among members.

Only 1 other $\beta$ Pic star observed has no previously reported EW[Li] value, HD 15115, for which we measure a EW of $0.10 \pm 0.02$. However, with a spectral type of F8, this measurement does not significantly constrain the star's age, given the slow decrease in lithium abundance with time for F stars (Mamajek \& Hillenbrand 2008). We note that HD 15115 is the lowest probability member (at 60\%) listed in Torres et al. (2008).

TYC 1752-63-1 was recently proposed by Schlieder et al. (2010) to be a member of the AB Dor Moving Group. This star exhibits $\mathrm{H} \alpha$ emission and lithium absorption, both of which strengthen the case of an adolescent age and association with the AB Dor Moving Group.

\subsection{F, G, and K Spectral Types}

Measured ratios of temperature sensitive absorption lines in the spectra of Sun-like stars are a powerful method of determining stellar effective temperature (e.g. Strassmeier \& Fekel et al. 1990). In principle this is accomplished best by measuring two absorption features of a particular element with substantially different excitation energies. In practice, however, the success of this relies upon identifying features that can be measured precisely, given the spectral resolution and wavelength coverage, and then being able to calibrate these ratios using spectra of stars with accurately determined temperatures. To investigate the possibility of this with our spectra, we searched by eye for absorption features within $\sim 10 \AA$ of each other that appear to vary inversely with temperature. The temperature dependence is established from our DeVeny observations of stars in the study of Valenti \& Fischer (2005), who determine effective temperatures for F-, G- and K-stars accurate to $44 \mathrm{~K}$ via comparisons with synthetic stellar spectra. The EWs of these features are determined by fitting a Gaussian profile to the absorption feature using tools in IRAF. The 3 line pair ratios that show the strongest correlation between EW ratios and temperature and that we adopt for temperature determination in this study are 6137/6142 $\AA, 6162 / 6170 \AA$, and 6450/6456 .

To better understand the substratum of the temperature sensitivity of these line ratios, 
we referred to the high resolution $(\mathrm{R} \sim 350,000)$ spectrum of the Sun provided by Lobel (2007), who label solar absorption features and provide excitation energies. From this we determine that the line ratio 6137/6142 actually consist of Fe I 6136.615 (2.453 eV), Fe I $6136.993(2.198 \mathrm{eV})$ and Fe I $6137.694(2.588 \mathrm{eV})$ versus Ba II $6141.713(0.704 \mathrm{eV})$ and Fe I $6141.730(3.603 \mathrm{eV})$; excitation energies are given in parentheses. The line ratio 6162/6170 consists of Ca I 6162.173 (1.899 eV) versus Ca I $6169.042(2.527 \mathrm{eV}) \mathrm{Ca}$ I 6169.563 (2.526 eV) and Fe I $6170.504(4.795 \mathrm{eV})$. And finally, the line ratio 6450/6456 consists of Ca I 6449.808 $(2.521 \mathrm{eV})$ versus Ca I $6455.598(2.523 \mathrm{eV})$ and Fe II $6456.383(3.903 \mathrm{eV})$. The temperature sensitivity in these cases likely stems from the individual features with the most discrepant excitation energies. We also note that all of these blends are unresolved in our modest resolution spectra as determined by the absence of any significant residuals of the best fit Gaussians.

To quantify the temperature dependence of these line ratios, a linear trend is fit to the line ratios versus temperature; higher order fits appear unwarranted. The best fit linear relations are shown in Figure 2, Ten Valenti \& Fischer (2005) stars are used to determine the best fit relation for the $6137 / 6142$ and $6162 / 6170$ ratios, while 15 stars are used to determine the 6450/6456 ratio; as noted in Section 3, stars observed in the May run had a slightly redder wavelength setting, and thus did not contain the two bluer line ratios. Valenti \& Fischer (2005) estimate metallicities for all of the stars used in these calibrations, and although most have metallicity estimates within \pm 0.12 of solar, two stars are slightly metal rich, HD $182488([\mathrm{Fe} / \mathrm{H}]=+0.22)$ and $\mathrm{HD} 145675([\mathrm{Fe} / \mathrm{H}]=+0.46)$. However, the the best fit relations predict temperatures based on their measured ratios that are within 20 $\mathrm{K}$ of their independently determined values. Thus, there is no evidence that these relations are strongly metal dependent.

There are 2 primary sources of error inherent to this method for estimating temperature. The first stems from how well the standard stars agree with one another. We estimate this from the $1 \sigma$ dispersion about the best fit linear relations illustrated in Figure 2, which are $114 \mathrm{~K}, 182 \mathrm{~K}$, and $152 \mathrm{~K}$, respectively. Since the temperature is determined by equally weighting all 3 line ratio estimates, we estimate the combined error by averaging these 3 dispersion estimates and dividing by $\sqrt{3}$. This yields a typical uncertainty of $86 \mathrm{~K}$. The second source of error stems from our ability to measure the EWs of young stars due to the noise in the spectra. The typical EW uncertainty of $\pm 0.01 \AA$ corresponds to temperature uncertainty of $\sim 60 \mathrm{~K}$. We add these 2 error terms in quadrature and assign temperature uncertainties of $105 \mathrm{~K}$ using this technique.

We used this method to estimate temperature estimates for the F-, G-, and early-K stars $\left(T_{\text {eff }}\right.$ ranging from $4600-6300 \mathrm{~K}$ ) in the observed sample, which is 11 of the 19 stars 
observed. For all but one star (PX Vir), the temperature assignments are an average of all 3 EW ratio estimates. Because PX Vir was observed only in May, the spectral range does not contain the features from the first two ratios. In this case, the composition of this star's temperature is solely determined from the 6450/6456 EW ratio, and has a larger uncertainty of $163 \mathrm{~K}$. We also note that PX Vir is spectroscopic binary, and its companion star may introduce a systematic error not accounted for in our temperature estimates. The results of all temperature determinations are listed in Tables 1 and 2 .

We compare these newly determined temperatures to temperatures inferred from the previously assigned spectral types listed in Tables 1 and 2. The previous spectral types originate from a variety of techniques, but most are determined via comparisons with optical spectra, similar to the method described in Section 4.4. We assign temperatures to these spectral types using the spectral type relationship in Hartigan et al. (1994). All but 2 stars have temperatures that are consistent to within $300 \mathrm{~K}$, or effectively 2 spectral subclasses. The 2 exceptions are the F8 stars HD 14082 AB and HD 15115, previously classified as F5 and F4, respectively. We note however that HD 15115 is included in the Valenti \& Fischer (2005) spectral synthesis study, which we used to calibrate our temperature relations 4.3, and they determine a temperature of $6219 \mathrm{~K}$, consistent with our determined temperature of $6120 \pm 105 \mathrm{~K}$. Our technique is at least self-consistent and the apparent discrepancy may stem in part from the temperature/spectral type relation adopted for these comparisons.

\subsection{Late-K and M Spectral Types}

For stars cool enough to be classified as late-K and $\mathrm{M}$, their spectra transition from being dominated by atomic features to being dominated by molecular band features which greatly inhibits the use of atomic features to determine temperature. For these stars we rely upon direct comparisons with stars of known spectral type and then adopt a spectral type temperature scale to estimate temperatures.

Our spectral comparisons rely upon comparisons of newly obtained DeVeny spectra of stars classified as 'primary standards' in Kirkpatrick et al. (1991). These types of comparisons are well known to yield spectral types accurate to at least 0.5 subclasses, given the strong variations of the TiO bands with temperature (e.g. Webb et al. 1999). Because we were not able to obtain comparison standards at all $\mathrm{M}$ spectral subclasses, we approximate intermediate subclasses in some cases by averaging cooler and hotter spectra together (e.g. averaging an M3 and an M4 to approximate an M3.5). Figure 3 illustrates several examples of these comparisons for 4 Moving Group $M$ dwarfs. Based on the rapid changes in the spectra with temperature, spectral type uncertainties are 0.5 spectral subclasses. Out of the 
19 observed Moving Group members, 8 spectral types are determined this way. These newly assigned spectral types are consistent with the ones assembled in Torres et al. (2008) to 0.5 spectral subclasses, with no systematic difference.

The effective temperatures assigned to the 8 late $K$ and $M$ dwarf stars in this study are set using the spectral type/temperature scale from Hartigan et al. (1994), which is appropriate for dwarf stars. While there is evidence that very young ( few Myr) M stars have a temperature scale that is warmer than this, given their slightly extended atmosphere (White et al. 1999; Luhman et al. 2003), there is no convincing evidence that this offset persists for many tens of millions of years. The uncertainty of 0.5 spectral subclass corresponds to a temperature uncertainty $\sim 75 \mathrm{~K}$.

\section{Angular Size Estimates}

In this section we estimate angular sizes for the $127 \mathrm{AB}$ Dor and $77 \beta$ Pic members assembled in Tables 1 and 2. This is accomplished by using the assembled temperatures along with estimated luminosities to calculate stellar radii, that in combination with the measured or estimated distances, can predict angular sizes. The assembled temperatures and distances are described in Section 2. Luminosities are determined by correcting the apparent $V$-band magnitudes to an absolute magnitude, and then to a bolometric luminosity using bolometric corrections from Hartigan et al. (1994) for F-type stars and values from Kenyon \& Hartmann (1995) for A and B stars. We adopt a solar bolometric luminosity of $M_{\text {bol }}(\odot)=4.83$ in these calculations (Glushneva et al. 2002). The bolometric corrections are done relative to the $V$-band since the median spectral type of the sample is late-G, which has an energy distribution that peaks at optical wavelengths.

Of the 204 stars listed as potential members of the AB Dor and $\beta$ Pic Moving Groups, we are able to estimate angular sizes for 167 stars (Tables 1 and 2). The 37 stars for which we do not estimate sizes are in most cases companion stars that are too close to their primary to have spatially resolved photometry and/or a spectral type. This close proximity makes them less ideal targets for interferometric measurements in any case. Twenty-five of the sizes estimated are of multiple star systems with spatially unresolved $V$-band magnitudes. This subset of size estimates, which are biased toward larger values, are indicated in Tables 1 and 2 with brackets. The estimated angular sizes range from 0.06 mas to 1.17 mas. The 5 largest stars are the B6 star $\alpha$ Gru (1.17 mas), the M1 star AU Mic (0.72 mas), the A3 star $\beta$ Pic (0.69 mas), the M2.5 star GJ 393 (0.69 mas), and the F8 star HD 25457 (0.63 mas); all five are single stars. 
As a check on our prescription for calculating angular sizes, we apply the same methodology to predict the angular diameters of a sample of stars whose diameters have been directly measured via interferometry. Stars were selected from van Belle \& von Braun et al. (2009), who report new measurements from the Palomar Testbed Interferometer (Colavita et al. 1999) and assemble previous CHARA Array measurements from Baines et al. (2008); both data sets report angular sizes accurate to a few percent. The comparison sample includes late-B through early-M main sequence stars; we use the spectral types, $V$ magnitudes, and distances provided in their study. The average difference between the measured and calculated sizes is $-3 \%$, although with a large dispersion of $21 \%$. However, many of the stars with the largest discrepancies have temperatures assigned from spectral types that are vastly different $(\geq 380 \mathrm{~K})$ from the values inferred directly from interferometric radius measurements. The most extreme case is the HD 157881, which has an interferometrically determined temperature of $3664 \mathrm{~K}$, but an adopted K2 temperature of $4900 \mathrm{~K}$. In many of these cases we suspect large spectral type errors; van Belle \& von Braun (2009) did not redetermined spectral types, but assembled them from uncited sources. If we restrict the sample to the 31 stars whose interferometrically determined temperatures that agree with those from their spectral type to better than $380 \mathrm{~K}$ (corresponding to approximately \pm 2 spectral subclasses) and include only dwarf stars, the average difference between the measured and calculated sizes reduces to $-2 \%$ with a dispersion of $8 \%$. This result is also corroborated with a comparison of the subset of radii measurements that have uniformly determined spectral types from Gray et al. (2001) and Gray et al. (2003). For this subset of 19 stars, the average difference between the measured and calculated sizes is $-5 \%$ with a dispersion of $10 \%$. Since the spectral types of many of these newly identified Moving Group members are determined from modern prescriptions, such as that outlined in Sections 4.2 and 4.3, with temperature errors of less than a few hundred Kelvin, we adopt $8 \%$ as the typical size uncertainty for the predicted sizes for stars with Hipparcos determined distances.

\section{Spatially Resolvable Young Stars}

In Figure 4 we illustrate the angular diameters calculated for 167 of the 204 Moving Group members, all of which have $B_{T}-V_{T}$ color, $K$ magnitude, declination, and angular size estimates; one star ( $\alpha$ Gru, angular diameter $=1.17$ mas) has a diameter larger than the range of this plot. We distinguish subsets of this sample based on those that are likely large enough and bright enough to be spatially resolved by long-baseline optical/infrared interferometer. We set a size of 0.4 mas as the minimum size to be spatially resolved, consistent with what has been demonstrated with interferometric baselines larger than $300 \mathrm{~m}$ (e.g. Baines et al. 2007). We use the $K_{2 M A S S}$ magnitudes to assess whether the stars are bright 
enough to observe, adopting 7.0 as the practical magnitude limit of operational interferometers. However, of the 167 stars for which we have size estimates, only 159 have $K_{2 M A S S}$ magnitudes; as noted in Section 2, fewer pairs have spatially resolved 2MASS measurements than Tycho II measurements. For these 8 stars with missing 2MASS measurements, we estimate their $K_{2 M A S S}$ magnitudes using the resolved $V$ magnitude, spectral type, and the $V-K$ color relations of Hartigan et al. (1994) and Kenyon \& Hartmann (1995); we do not list these approximate $K$ magnitudes in the Tables.

Of the 167 Moving Group members with size estimates and $K_{2 M A S S}$ photometry, 18 are large enough $\left(>0.4\right.$ mas) and bright enough $\left(K_{2 M A S S}<7.0\right)$ to be observed and spatially resolved. However, only 6 stars are north enough $(\mathrm{DEC}>-30)$ to be easily accessible to northern interferometers, which currently has the longest baseline interferometers. These 6 include include the $\beta$ Pic stars 51 Eri (F0; 0.52 mas) and AF Lep A (F7; 0.40 mas) and the AB Dor stars HD 17573 A (B8; 0.56 mas), HD 25457 (F8; 0.63 mas), GJ 393 (M2.5; $0.69 \mathrm{mas}$ ), and $\delta \mathrm{Scl}$ (A0; $0.44 \mathrm{mas})$. Of these, GJ 393 is of particular interest. While the intermediate mass F, A and B stars should all be zero-age main sequence stars for even the youngest ages proposed for these associations, GJ 393, with a spectral type of M2.5, is not predicted to achieve this until an age of $\sim 100$ Myr (e.g. Baraffe et al. 1998; Siess et al. 2000). Thus, depending upon the age of AB Dor, GJ 393 could be distinctively pre-main sequence and its location above the main sequence could help to constrain the age of this Moving Group.

Although all 18 stars are accessible to southern interferometers (DEC $<+30$ ), the shorter baselines of these facilities restrict the sample resolvable to only the B6 star $\alpha$ Gru (1.17 mas). We nevertheless highlight that if resolutions comparable to CHARA are achievable, many stars could be spatially resolved since the majority of Moving Group members are in the southern hemisphere sky. Two high-profile examples include the debris disk hosting M dwarf AU Mic (0.72 mas) and the young solar analogue AK Pic A (0.48 mas). Size measurements of these stars would better constrain their ages and the age (and possibly age spread) of the Moving Group. Moreover, these temporal stamps would help trace the rate of disk evolution and possibly planetary system formation.

\section{Summary and Comments on Future Prospects}

We present moderate resolution $(\mathrm{R} \sim 3575)$ optical spectra of 19 members of the $\mathrm{AB}$ Dor and $\beta$ Pic Moving Groups, including 5 recently proposed members. The strengths of $\mathrm{H} \alpha$ emission and LiI $6708 \AA$ absorption, both signatures of youth, are extracted from these spectra. The detection of Li I $6708 \AA$ in the proposed $\beta$ Pic members TYC 1186-706-1 (K7) 
and TYC 7443-1102-1 (K7.5) further strengthen the case for youth and membership within this young Moving Group. No Li I is detected in the spectrum of the proposed $\beta$ Pic member TYC 2211-1309-1 (K7). Although this alone can not refute its membership given the spread in Li I abundances for known members (Mentuch et al. 2008; da Silva et al. 2009), it does suggest it is slightly older than other known members; it would be the only $\beta$ Pic star of $\mathrm{K}$ spectral type without detected lithium. Li I is also detected in the spectrum of the recently proposed member of the AB Dor Moving Group, TYC 1752-63-1 (K5), which likewise strengthens its case for membership. In addition to these measurements, temperature sensitive line ratios are used to estimate the temperatures of $\mathrm{F}$ through early- $\mathrm{K}$ stars, and direct comparisons with spectral standards are used to determine spectral types for late-K and M stars.

Updated membership lists for both Moving Groups are assembled with an emphasis placed on identifying multiple star system and spatially resolved photometric measurements. Currently, the AB Dor moving group contains 127 proposed members and the /beta Pic moving group holds 77 proposed members. For these ensemble samples, temperatures determined from either new or previous measurements are used in combination with distances and Tycho II photometry to predict angular sizes of 167 proposed members. A comparison of sizes predicted in this way for a sample of main sequence stars that have been spatially resolved interferometrically implies that the size estimates are accurate to $8 \%$. Six of these Moving Group members are bright enough $(K<7.0)$, large enough $\left(\theta_{\text {res }}>0.4\right.$ mas $)$ and north enough (DEC > -30) to be spatially resolvable with the CHARA Array, the world's longest baseline interferometer working at optical/infrared wavelengths. One of these stars is the low mass AB Dor member GJ 393; size measurements of this likely pre-main sequence star could help constrain the age of AB Dor, which is somewhat poorly determined. Using the same brightness and resolution criteria, 18 stars could be observed from the southern hemisphere (DEC $<+30$; includes all 6 northernly accessible stars). However, the operational baselines of southern hemisphere interferometers currently will only allow the largest of these, the B6 star $\alpha$ Gru (1.17 mas), to be spatially resolved.

Dramatically increasing the number of young stars that can be spatially resolved interferometrically would not only improve age estimates for these associations, but would also provide powerful constraints on the early evolution of young stars settling onto the main sequence, over a range of masses and with a range of rotational velocities. This will require improving the effective resolution limit of interferometers, since the vast majority of young stars have sizes smaller than 0.4 mas (Figure 4). Here we comment on the most practical way to achieve this with an emphasis on the lowest mass stars that, according to evolutionary models, should still pre-main sequence. 
Interferometers are able to determine the angular size of a spatially resolved source by measuring its reduced fringe contrast, or visibility, relative to that of an unresolved source. For a perfectly calibrated system, unresolved sources have a visibility of 1.0 while resolved sources have a visibility less than this (see Haniff 2007, for a review). Although the smallest angular size that an interferometer can measure is typically approximated as $\lambda /(2 \times$ baseline $)$, which corresponds to 0.5 mas for $\mathrm{H}$-band observations at a baseline of $331 \mathrm{~m}$, in practice the true limit is set by how accurately the visibility can be measured; reductions in the visibility below 1.0 can also be caused by instrumental and/or environmental effects. These effects are typically accounted for by observing spatially unresolved stars to calibrate the system's visibility (e.g. Boden 2007). However, turbulence in the atmosphere and vibrations along the optical path make these calibrations imperfect. Facilities that spatially filter the light from each telescope (e.g. Coudé du Foresto et al. 1997) or that utilize adaptive optics systems on their telescopes (e.g. Wizinowich 2006) appear to successfully mitigate the former, while improved metrology systems may help mitigate the latter (e.g. Wyant 2002). Meanwhile, more precisely determined visibilities could be obtained simply by greatly increasing the number of observations. While we can not make specific recommendations on how to achieve this, we highlight that more robustly determined visibility measurements is one practical way of improving the effective resolution of current interferometers, thereby allowing more young stars to be spatially resolved.

A more direct way of increasing the number of stars that can be spatially resolved is to increase the baselines of either northern or southern facilities. For example, doubling the longest baselines to $\sim 660 \mathrm{~m}$ would improve the resolution to 0.2 mas, and allow 37 stars in the north and 65 stars in the south to be resolved (from Tables 1 and 2). Moreover, this would permit a large fraction of stars in other Moving Groups (e.g. TW Hydrae) to also be resolved. Although in principle this only requires 1 additional telescope and in many cases is logistically feasible, this does raise an additional issue for visibility calibration. As noted above, an important part of accurate size measurements is observations of unresolved stars (point sources) to calibrate the visibilities. However, at baselines of $660 \mathrm{~m}$, the majority of stars brighter than $K=7$ are spatially resolved, making them somewhat poor stars for visibility calibration. Thus, to be fully realizable, this solution requires not only the addition of telescopes to extend the baseline, but either larger telescopes or a dramatic improvement in sensitivity that permit observations of fainter, smaller stars that are spatially unresolved.

An alternative to increasing the baseline to improve resolution is to observe at shorter (e.g. optical) wavelengths. Several facilities already operate in the $V, R$, and $I$ bands, which offer resolution improvements by factors of 2-4 relative to $H$ - or $K$-band. If we assume an optical limiting magnitude of $V=7$ and a resolution limit of 0.2 mas, $20 \mathrm{AB}$ Dor and $\beta$ Pic stars could be spatially resolved, 9 accessible from northern facilities (DEC $>-30)$ and 21 
accessible from southern facilities (DEC $<+30$ ). However, of these 20 stars, 16 have $\mathrm{B}, \mathrm{A}$ or F spectral types; the 4 remaining stars are classified as G (HD 45270, AK Pic A, V824 Ara A) or early K (AB Dor A) spectral type. We note this because while this option is likely to be realizable soon, it will only permit Sun-like (or larger) stars to be observed which reach the main sequence in $\sim 30$ Myr or less. Resolving a large number of bona-fide pre-main sequence stars will require a sensitivity to spectral type $\mathrm{M}$ stars, with a characteristic $V$ magnitude of 11. This is again likely only achievable with larger telescopes or systems with dramatically improved sensitivity.

We are grateful for the observational assistance provided by Tom Bida and thank A. Reidel and T. Henry for their help in acquiring DeVeny spectra and for insightful comments during the inception of this project. We also thank M. Simon and our astute referee for helpful comments on the first version of this manuscript. This project was funded by NSF/AAG grant \# 0908018 and a GSU Research Initiation Grant.

\section{REFERENCES}

Bailey, J. I., III, White, R. J., Blake, C. H., Charbonneau, D., Barman, T., Tanner, A. M. \& Torres, G. 2011, ApJ, submitted

Baines, E. K., van Belle, G. T., ten Brummelaar, T. A., McAlister, H. A., Swain, M., Turner, N. H., Sturmann, L., \& Sturmann, J. 2007, ApJ, 661, 195

Baines, E. K., McAlister,H. A., ten Brummelaar, T. A., Turner,N. H., Sturmann, J., Sturmann, L., Goldfinger, P. J., \& Ridgway, S. T. 2008a, ApJ, 680, 728

Baines, E. K., et al. 2010, ApJ, 710, 1365

Baraffe, I., Chabrier, G., Allard, F., \& Hauschildt, P. H. 1998, A\&A, 337, 403

Baraffe, I., Chabrier, G., Allard, F., \& Hauschildt, P. H. 2002, A\&A, 382, 563

Bessell, M. S. 2000, PASP, 112, 773

Biller, B. A., et al. 2007, ApJ, 173, 143

Biller, B. A., et al. 2010, ApJ, 720, 82

Bodenheimer, P. 1965, ApJ, 142, 451

Boden, A. F. 2007, New Astronomy Reviews, 51, 617 
Boyajian, T. S., et al. 2008, ApJ, 683, 424

Colavita, M. M., et al. 1999, ApJ, 510, 505

Collier-Cameron, C. A., \& Foing, B. H. 1997, Observatory, 117, 218

Coudé du Foresto, V., Ridgway, S., \& Mariotti, J. M. 1997, A\&A, 121, 379

Close, L., M., et al. 2005, Nature, 433, 286

Covino, E., Alcala, J. M., Allain, S., Bouvier, J., Terranegra, L., \& Krautter, J. 1997, A\&A, 328,187

Craig, N., Christian, D. J., Dupuis, J., \& Roberts, B. A. 1997, AJ, 114, 244

da Silva, L., Torres, C. A. O., de La Reza, R., Quast, G. R., Melo, C. H. F., Sterzik, M. F. 2009, A\&A, 508, 833

Davis, J., Tango, W. J., Booth, A. J., ten Brummelaar, T. A., Minard, R. A., \& Owens, S. M. 1999, MNRAS, 303, 703

Evans, T. M., et al. 2012, ApJ, accepted

Feigelson, E. D., Lawson, W. A., Stark, M., Townsley, L., \& Garmire, G. P. 2006, AJ, 131, 1730

Glushneva, I. N., Shenavrin, V. I., \& Roshchina, I. A. 2002, Astronomical and Astrophysical Transactions, 21, 317

Gray, R. O., Napier, M. G., \& Winkler, L. I. 2001, AJ, 121, 2148

Gray, R. O., Corbally, C. J., Garrison, R. F., McFadden, M. T., \& Robinson, P. E. 2003, AJ, 126, 2048

Hanbury Brown, R., Davis, J., Lake, R. J. W., \& Thompson, R. J. 1974, MNRAS, 167, 475

Haniff, C. 2007, New Astronomy Reviews, 51, 583

Hartigan, P., Strom, K. M., \& Strom, S. E. 1994, ApJ, 427, 961

Hillenbrand, L. A., \& White, R. J. 2004, ApJ, 604, 741

$\mathrm{H} ø \mathrm{~g}$, E., et al. 2000, A\&A, 355, 27

Hormuth, F., Brandner, W., Hippler, S., Janson, M., \& Henning, T. 2007, A\&A, 463, 707 
Hummel, C. A., et al. 2003, AJ, 125, 2630

Janson, M., et al. 2007, A\&A, 462, 615

Kasper, M., Apai, D., Janson, M., \& Brandner, W. 2007, A\&A, 472, 321

Kenyon, S. J., \& Hartmann, L. 1995, ApJS, 101, 117

Kirkpatrick, J. D., Henry, T. J., \& McCarthy, D. W. 1991, ApJS, 77, 417

Kiss, L. L., et al. 2011, MNRAS, 411, 117

Labeyrie, A. 1975, ApJ, 196, 71

Lépine, S., \& Simon, M. 2009, AJ, 137, 3632

Lobel, A. 2007, SpectroWeb: An Interactive Graphical Database of Digital Stellar Spectral Atlases, in "The Ultraviolet Universe: Stars from Birth to Death", 26th meeting of the IAU, Editorial Complutense Univ. of Madrid, ed. A. Gomez de Castro and M. Barstow, 167, Aug 2006, Prague, Czech Republic.

López-Santiago, J., Montes, D., Crespo-Chacn, I., \& Fernndez-Figueroa, M. J. 2006, ApJ, 643,1160

Lowrance, P., et al. 2000, ApJ, 541, 390

Luhman, K. L., Stauffer, J. R., Muench, A. A., Rieke, G. H., Lada, E. A., Bouvier, J., \& Lada, C. J. 2003, ApJ, 593, 1093

Luhman, K. L., Stauffer, J. R., \& Mamajek, E. E. 2005, ApJ, 628, 69

Mamajek, E. E., \& Hillenbrand, L. A. 2008, ApJ, 687, 1264

Mathieu, R. D., Baraffe, I., Simon, M., Stassun, K. G., \& White, R. 2007 University of Arizona Press, Tucson, 951, 411

Macdonald, J., \& Mullan, D. J. 2010, ApJ, 723, 1599

McAlister, H., Hartkopf, W. I., \& Franz, O. G. 1990, AJ, 99, 965

Mentuch, E., Brandeker, A., van Kerkwijk, M. H., Jayawardhana, R., \& Hauschildt, P. H. 2008, ApJ, 689, 1127

Michelson, A. A., \& Pease, F. G. 1921, ApJ, 53, 249 
Montes, D., Lpez-Santiago, J., Gálvez, M. C., Fernández-Figueroa, M. J., De Castro, E. \& Cornide, M. 2001, MNRAS, 328, 48

Moor, A., Abraham, P., Derekas, A., Kiss, C., Kiss, L. L., Apai, D., Grady, C., \& Henning, T. 2006, ApJ, 644, 525

Morlet, G., Salaman, M., \& Gili, R. 2000, A\&A, 145, 67

Mourard, D., et al. 2010, A\&A, 508, 1073

Nielson, E. L., \& Close, L. M. 2010, ApJ, 717, 878

Ortega, V. G., Jilinski, E., de La Reza, R., \& Bazzanella, B. 2007, MNRAS, 377, 441

Perryman, M. A. C., et al. 2009, A\&A, 500, 501

Petrov, R. G., et al. 2007, A\&A, 464, 1

Raghavan, D., et al. 2010, ApJ, 190, 1

Schlieder, J. E., Lépine, S., \& Simon, M. 2010, AJ, 140, 119

Siess, L., Dufour, E., \& Forestini, M. 2000, A\&A, 258, 593

Skrutskie, M.F., et al. 2006, AJ, 131, 1163

Song, I., Zuckerman, B., \& Bessell, M. S. 2003, ApJ, 599, 342

Strassmeier, K. G., \& Fekel, F. C. 1990, A\&A, 230, 389

ten Brummelaar, T.A., et al. 2005, AJ, 628, 453

Torres, C. A. O., Quast, G. R., da Silva, L., de La Reza, R., Melo, C. H. F., \& Sterzik, M. 2006, A\&A, 460, 695

Torres, C. A. O., Quast, G. R., Melo, C. H. F., \& Sterzik, M. F. 2008, Handbook of Star Forming Regions, Volume II: The Southern Sky ASP Monograph Publications, 5, 757

Valenti, J. A., \& Fischer, D. A. 2005, ApJS, 159, 141

van Belle, G. T., \& von Braun, K. 2009, ApJ, 694, 1085

van Leeuwen, F. 2007, A\&A, 474, 653

Wahhaj, Z., et al. 2011, ApJ, 729, 139 
Webb, R. A., Zuckerman, B., Platais, I., Patience, J., White, R. J., Schwartz, M. J., \& McCarthy, C. 1999, ApJ, 512, 63

Weise, P., Launhardt, R., Setiawan, J., \& Henning, T. 2010, A\&A, 517, 88

West, A., et al. 2008, AJ, 135, 785

White, R. J., Ghez, A. M., Reid, I. N., \& Schultz, G. 1999, ApJ, 520, 811

Wizinowich, P. L. 2006, SPIE, 6034, 1

Wyant, J. C. 2002, SPIE, 4927, 154

Yee, J. C., \& Jensen, E. L. N. 2010, ApJ, 711, 303

Young, P. A., \& Arnett, D. 2005, ApJ, 618, 908

Zickgraf, F. J., Krautter, J., Reffert, S., Alcalá, J. M., Mujica, R., Covino, E., \& Sterzik, M. F. 2005, A\&A, 433, 151

Zuckerman, B., Song, I., Bessell, M. S., \& Webb, R. A. 2001, ApJ, 562, 87

Zuckerman, B., \& Song, I. 2004a, ARA\&A, 42, 685

Zuckerman, B., Song, Inseok, \& Bessell, M. S. 2004b, ApJ, 613, 65

Zuckerman, B., Rhee, J. H., Song, I., \& Bessell, M. S. 2011, ApJ, 732, 61 

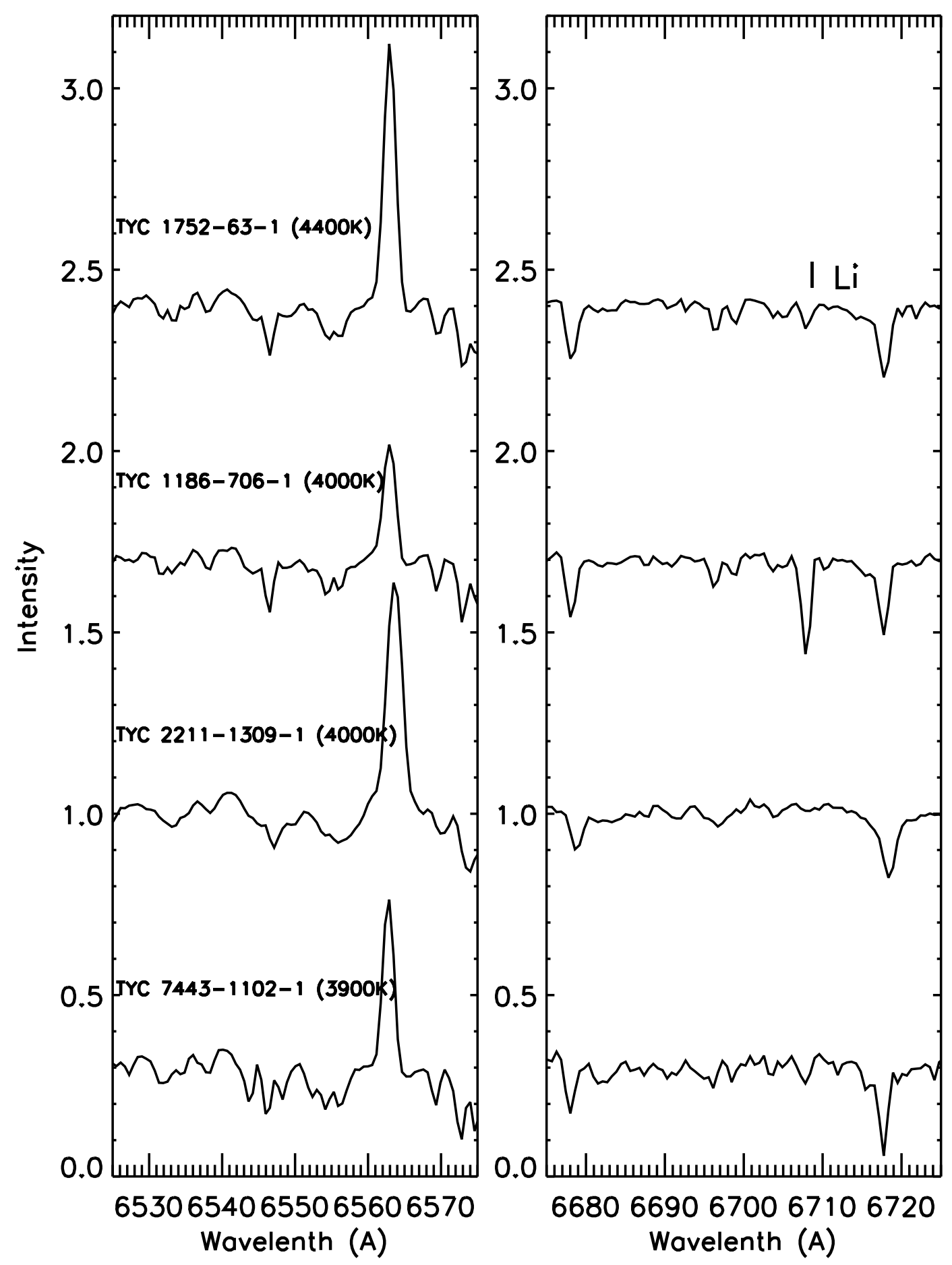

Fig. 1.- Portions of DeVeny specrta of 4 Moving Group stars. The left panel shows portions near the $\mathrm{H} \alpha$ emission feature (left panel) and the Li I $6708 \AA$ absorption feature (right panel). Labels give the names and determined temperatures. Lithium is detected in 3 of these 4 stars; the exception is the K7 star TYC 2211-1309-1. 

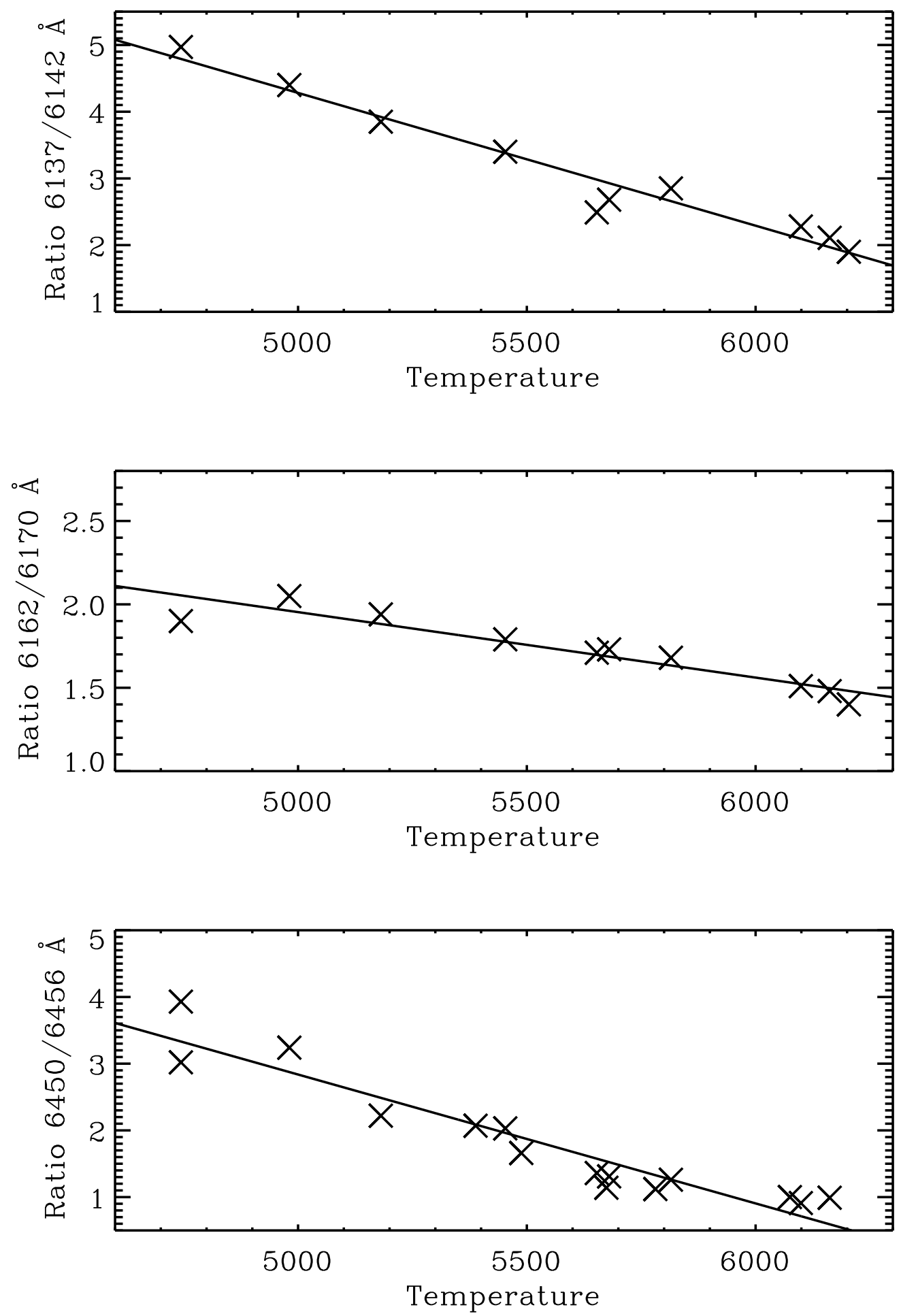

Fig. 2.- Equivalent width ratios versus temperature for stars with temperatures determined by Valenti \& Fischer (2005). The solid lines illustrate the best fit linear relations used to estimate temperatures for the observed Moving Group stars. From top to bottom, the dispersions about this best fit, a measure of uncertainty, are $114 \mathrm{~K}, 182 \mathrm{~K}$, and $152 \mathrm{~K}$, respectively. 

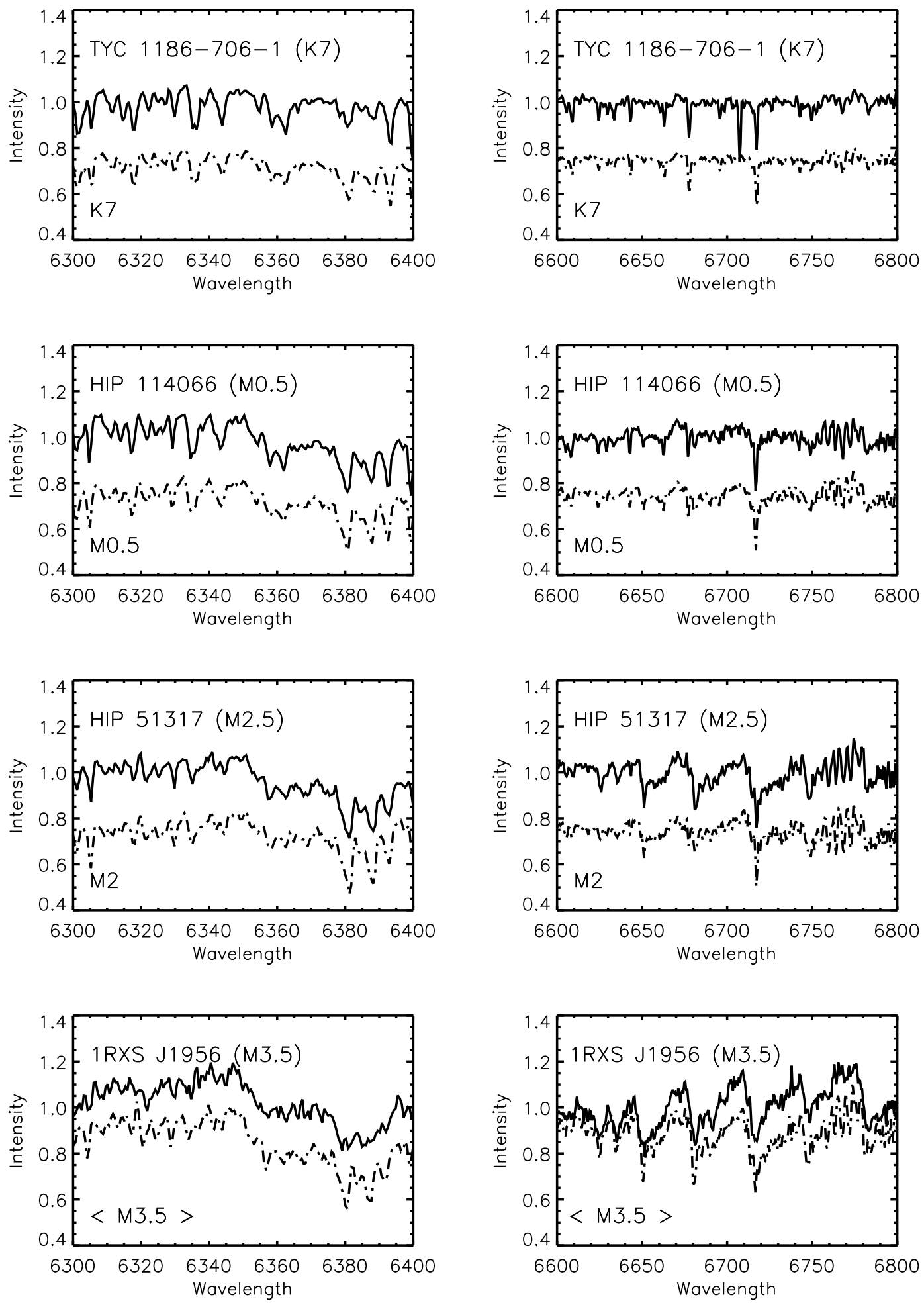

Fig. 3.- Illustration of the method used to determine spectral types for late-K and -M stars. Spectral types are assigned to the observed spectra (solid lines) based on the best matching spectral standard (dot-dashed lines, labeled) or spectral standard average (labeled in brackets). 

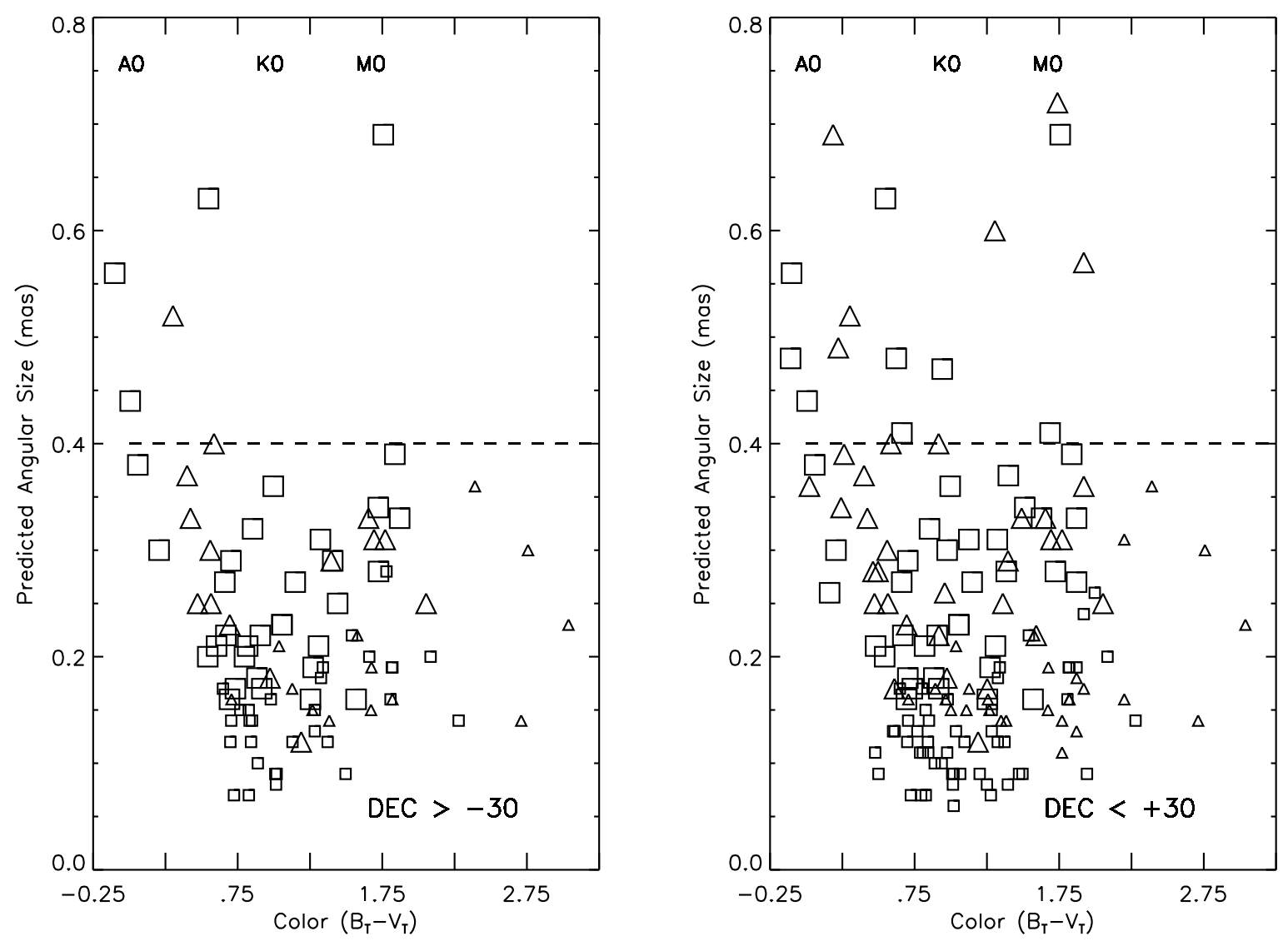

Fig. 4.- Angular diameter versus Tycho $B_{T}-V_{T}$ color for AB Dor stars (squares) and $\beta$ Pic stars (triangles). The left panel displays stars visible in the northern hemisphere (Dec $>-30)$ while the right panel shows stars visible from the south $($ Dec $<+30)$. One proposed member of AB Dor, $\alpha$ Gru with a size of 1.17 mas is above the size range illustrated; at a declination of -47 , it is only accessible to southern facilities. The dashed lines represents a characteristic resolution limit for current long-baseline interferometric facilities. Large symbols represent infrared bright stars $(K<7.0)$. According to these criteria, there are 18 stars that are bright enough and large enough to observe in the southern hemisphere but only 6 such stars in the north (see Tables 1 and 2). 
Table 1. Proposed AB Doradus Moving Group Members

\begin{tabular}{|c|c|c|c|c|c|c|c|c|c|c|c|c|}
\hline HD & HIP & Other & $\begin{array}{c}\operatorname{Dist}^{a} \\
(\mathrm{pc})\end{array}$ & $\begin{array}{c}V^{b} \\
\mathrm{mag}\end{array}$ & $\begin{array}{c}K^{b} \\
\mathrm{mag}\end{array}$ & $\begin{array}{c}\text { Prev. }^{c} \\
\text { SpT } \\
\end{array}$ & $\begin{array}{l}T_{\text {eff }} \\
(\mathrm{K})\end{array}$ & $\begin{array}{c}\text { New } \\
\text { SpT, } T_{\text {eff }}\end{array}$ & $\begin{array}{c}\operatorname{Lum}^{b} \\
\left(L_{\odot}\right)\end{array}$ & $\begin{array}{c}\theta^{b} \\
(\mathrm{mas}) \\
\end{array}$ & $\begin{array}{c}\text { Known }^{e} \\
\text { Mult }\end{array}$ & $\begin{array}{c}\text { Mult }^{f} \\
\text { Ref }\end{array}$ \\
\hline 1405 & $\cdots$ & PW And & 27 & 8.859 & 6.39 & $\mathrm{~K} 2$ & 4900 & $\cdots$ & 0.24 & 0.23 & $\mathrm{~S}$ & L07,NC10,E11 \\
\hline$\cdots$ & $\cdots$ & TYC 1741-2117-1 & 47.6 & 11.167 & 7.66 & $\mathrm{~K} 7$ & 4000 & $\cdots$ & 0.16 & 0.16 & $\ldots$ & $\cdots$ \\
\hline $4277 \mathrm{~A}$ & 3589 A & $\mathrm{BD}+54144 \mathrm{~A}$ & $48.6 \mathrm{H}$ & [7.803] & {$[6.36]$} & F8 & 6190 & $\cdots$ & [1.6] & {$[0.21]$} & B & E11 \\
\hline B & B & $\mathrm{BD}+54144 \mathrm{~B}$ & $48.6^{*}$ & $\cdots$ & $\cdots$ & $\cdots$ & $\cdots$ & $\ldots$ & $\ldots$ & $\cdots$ & В $3 . \prime 8$ & T08 \\
\hline 6569 & 5191 & BD-15 200 & $50.0 \mathrm{H}$ & 9.446 & 7.34 & K1 & 5080 & $\cdots$ & 0.45 & 0.16 & $\mathrm{~S}$ & E11 \\
\hline$\cdots$ & 6276 & BD-12 243 & $35.1 \mathrm{H}$ & 8.391 & 6.55 & G9 & 5410 & $\cdots$ & 0.53 & 0.22 & $\mathrm{~S}$ & E11 \\
\hline$\cdots$ & $\cdots$ & TYC 1752-63-1 & 38.5 & 10.723 & 7.64 & K5 & 4395 & $\mathrm{~K} 5,4400$ & 0.11 & 0.14 & $\ldots$ & $\ldots$ \\
\hline$\ldots$ & $\cdots$ & CD-46 644 A & 70 & 11.136 & {$[8.61]$} & K3 & 4730 & $\cdots$ & 0.21 & 0.09 & $\mathrm{~B}$ & $\cdots$ \\
\hline$\ldots$ & $\ldots$ & CD-46 $644 \mathrm{~B}$ & $70^{*}$ & $\ldots$ & $\ldots$ & $\ldots$ & $\ldots$ & $\ldots$ & $\ldots$ & $\ldots$ & B $21^{\prime \prime} 4$ & $2 \mathrm{M}$ \\
\hline 13482 & 10272 & $\mathrm{BD}+23296 \mathrm{~A}$ & $32.3 \mathrm{H}$ & 7.932 & {$[5.73]$} & K1 & 5090 & $\ldots$ & 0.74 & 0.32 & B & $\ldots$ \\
\hline B & $\mathrm{B}$ & $\mathrm{BD}+23296 \mathrm{~B}$ & $32.3^{*}$ & 9.519 & $\ldots$ & K4: & 4520 & $\cdots$ & 0.20 & 0.21 & B $1^{\prime \prime} 8$ & T08 \\
\hline $16760 \mathrm{Aa}$ & $12638 \mathrm{Aa}$ & $\mathrm{BD}+37604 \mathrm{Aa}$ & $50.2 \mathrm{H}$ & {$[8.764]$} & {$[7.03]$} & $\mathrm{G} 2$ & 5860 & $\ldots$ & {$[0.70]$} & {$[0.15]$} & $\mathrm{T}$ & E11 \\
\hline $16760 \mathrm{Ab}$ & $12638 \mathrm{Ab}$ & $\mathrm{BD}+37604 \mathrm{Ab}$ & $50.2^{*}$ & $\cdots$ & $\cdots$ & $\cdots$ & $\cdots$ & $\cdots$ & $\ldots$ & $\cdots$ & $\mathrm{T} 0 . ! 3$ & E11 \\
\hline B & $12635 \mathrm{~B}$ & $\mathrm{BD}+37604 \mathrm{~B}$ & $44.9 \mathrm{H}$ & 10.270 & 7.76 & $\mathrm{~K} 2$ & 4900 & $\ldots$ & 0.18 & 0.12 & $\mathrm{~T} 14^{\prime \prime} 6$ & 2M,E11 \\
\hline $17332 \mathrm{~A}$ & $13027 \mathrm{~A}$ & $\mathrm{BD}+18347 \mathrm{~A}$ & $32.6 \mathrm{H}$ & 7.446 & 5.52 & G1 & 5940 & $\cdots$ & 1.0 & 0.27 & $\mathrm{~B}$ & E11 \\
\hline B & $\mathrm{B}$ & $\mathrm{BD}+18347 \mathrm{~B}$ & $32.6^{*}$ & 8.170 & 5.64 & G6 & 5690 & $\cdots$ & 0.55 & 0.21 & В $3 . \prime 6$ & T08 \\
\hline $17573 \mathrm{~A}$ & $13209 \mathrm{~A}$ & $\mathrm{BD}+26471 \mathrm{~A}$ & $48.9 \mathrm{H}$ & [3.594] & {$[3.86]$} & B8 & 11900 & $\ldots$ & {$[160]$} & {$[0.56]$} & B & $\ldots$ \\
\hline $\mathrm{B}$ & B & $\mathrm{BD}+26471 \mathrm{~B}$ & $48.9^{*}$ & $\ldots$ & $\ldots$ & $\ldots$ & $\ldots$ & $\ldots$ & $\ldots$ & $\ldots$ & B $0 . ' 2$ & M90 \\
\hline 19668 & 14684 & IS Eri & $40.2 \mathrm{H}$ & 8.492 & 6.70 & G0 & 6030 & G5,5760 & 0.59 & 0.18 & $\mathrm{~S}$ & E11 \\
\hline$\ldots$ & 14809 & $\mathrm{BD}+21418 \mathrm{~A}$ & $49.4 \mathrm{H}$ & 8.513 & 6.97 & G5 & 5750 & G1,5970 & 0.87 & 0.16 & $\mathrm{~T}$ & E11 \\
\hline$\cdots$ & $14807 \mathrm{~A}$ & $\mathrm{BD}+21418 \mathrm{Ba}$ & $51.7 \mathrm{H}$ & [10.463] & {$[7.65]$} & K6 & 4260 & $\ldots$ & {$[0.32]$} & {$[0.18]$} & $\mathrm{T} 33^{\prime \prime} 1$ & 2M,E11 \\
\hline$\ldots$ & $14807 \mathrm{~B}$ & $\mathrm{BD}+21418 \mathrm{Bb}$ & $51.7^{*}$ & $\ldots$ & $\ldots$ & $\ldots$ & $\ldots$ & $\ldots$ & $\ldots$ & $\ldots$ & $\mathrm{T} 0^{\prime \prime} 3$ & $2 \mathrm{M}, \mathrm{E} 11$ \\
\hline$\ldots$ & $\ldots$ & HW Cet & $46 c$ & 10.270 & 8.02 & K4: & 4520 & $\ldots$ & 0.21 & 0.15 & $\ldots$ & $\ldots$ \\
\hline 20888 & 15353 & TYC 8866-1472-1 & $58.0 \mathrm{H}$ & 6.022 & 5.69 & A3 & 8720 & $\cdots$ & 13 & 0.26 & $\ldots$ & $\cdots$ \\
\hline $21845 \mathrm{~A}$ & $16563 \mathrm{~A}$ & V577 Per A & $33.8 \mathrm{H}$ & 8.250 & 6.37 & G5 & 5750 & $\cdots$ & 0.53 & 0.20 & $\mathrm{~B}$ & NC10,E11 \\
\hline B & B & V577 Per B & $33.8^{*}$ & 11.188 & 7.59 & M0 & 3800 & $\ldots$ & 0.10 & 0.20 & В $9 . \prime 5$ & 2M,E11 \\
\hline$\cdots$ & 17695 & RX J0347.3-0158 & $16.3 \mathrm{H}$ & 11.488 & 6.93 & M3 & 3350 & $\cdots$ & 0.036 & 0.33 & $\mathrm{~S}$ & L07,NC10,E11 \\
\hline 24681 & $\cdots$ & BD-02 754 & 53 & 9.042 & 7.25 & G8 & 5570 & $\ldots$ & 0.65 & 0.15 & $\ldots$ & $\ldots$ \\
\hline 25457 & 18859 & BD-00 632 & $19.2 \mathrm{H}$ & 5.378 & 4.18 & F6 & 6350 & $\mathrm{~F} 8,6200$ & 2.2 & 0.63 & $\mathrm{~S}$ & L07,NC10,E11 \\
\hline 25953 & 19183 & $\mathrm{BD}+01699$ & $55.3 \mathrm{H}$ & 7.825 & 6.58 & F5 & 6440 & $\mathrm{~F} 7,6250$ & 1.9 & 0.20 & $\mathrm{~S}$ & E11 \\
\hline$\cdots$ & $\cdots$ & TYC 0091-0082-1 & $37 \mathrm{c}$ & 10.931 & 8.65 & K0 & 5250 & $\cdots$ & 0.059 & 0.07 & $\cdots$ & $\cdots$ \\
\hline$\cdots$ & $\cdots$ & TYC 5899-26-1 & 16 & 11.632 & 6.89 & M3 & 3350 & $\cdots$ & 0.032 & 0.31 & $\cdots$ & $\cdots$ \\
\hline$\cdots$ & $22738 \mathrm{~A}$ & GJ $2036 \mathrm{~A}$ & $11.2 \mathrm{H}$ & 10.991 & 6.34 & M3 & 3350 & $\cdots$ & 0.027 & 0.41 & B & $\cdots$ \\
\hline$\ldots$ & B & GJ 2036 B & $11.2^{*}$ & 11.896 & 6.89 & M3 & 3350 & $\ldots$ & 0.012 & 0.27 & B $7{ }^{\prime \prime} 7$ & $2 \mathrm{M}$ \\
\hline
\end{tabular}


Table 1-Continued

\begin{tabular}{|c|c|c|c|c|c|c|c|c|c|c|c|c|}
\hline HD & HIP & Other & $\begin{array}{c}\text { Dist }^{a} \\
(\mathrm{pc})\end{array}$ & $\begin{array}{c}V^{b} \\
\operatorname{mag}\end{array}$ & $\begin{array}{c}K^{b} \\
\mathrm{mag}\end{array}$ & $\begin{array}{l}\text { Prev. }^{c} \\
\text { SpT }\end{array}$ & $\begin{array}{l}T_{\text {eff }} \\
(\mathrm{K})\end{array}$ & $\begin{array}{c}\text { New } \\
\text { SpT, } T_{\text {eff }}\end{array}$ & $\begin{array}{l}\operatorname{Lum}^{b} \\
\left(L_{\odot}\right)\end{array}$ & $\begin{array}{c}\theta^{b} \\
(\mathrm{mas})\end{array}$ & $\begin{array}{c}\text { Known } \\
\text { Mult }\end{array}$ & $\begin{array}{c}\text { Mult }^{f} \\
\text { Ref }\end{array}$ \\
\hline 31652 & $\ldots$ & BD-09 1034 & 88 & 9.981 & 8.35 & G8 & 5570 & $\ldots$ & 0.75 & 0.10 & $\ldots$ & $\ldots$ \\
\hline 32981 & $\ldots$ & BD-16 1042 & 81 & 9.119 & 7.74 & F9 & 6120 & $\ldots$ & 1.3 & 0.12 & $\ldots$ & $\ldots$ \\
\hline 293857 & $\ldots$ & BD-04 1063 & 78 & 9.244 & 7.36 & G8 & 5570 & $\ldots$ & 1.2 & 0.14 & $\ldots$ & $\ldots$ \\
\hline 33999 A & $\ldots$ & CD-34 $2128 \mathrm{~A}$ & 106 & {$[8.858]$} & {$[7.20]$} & F8 & 6190 & $\ldots$ & {$[2.8]$} & {$[0.13]$} & $\mathrm{T}$ & $\ldots$ \\
\hline 35650 & 25283 & CD-39 1951 & $17.7 \mathrm{H}$ & 9.144 & 5.92 & K6 & 4260 & $\ldots$ & 0.13 & 0.34 & $\ldots$ & $\ldots$ \\
\hline $36705 \mathrm{~A}$ & 25647 & AB Dor A & $14.9 \mathrm{H}$ & {$[6.938]$} & {$[4.69]$} & K0 & 5250 & $\ldots$ & {$[0.38]$} & {$[0.47]$} & $\mathrm{Q}$ & $\ldots$ \\
\hline $\mathrm{C}$ & $\mathrm{C}$ & AB Dor $C$ & $14.9^{*}$ & $\ldots$ & $\ldots$ & $\ldots$ & $\ldots$ & $\ldots$ & $\ldots$ & $\ldots$ & $\mathrm{Q} 0 . \prime 2$ & T08 \\
\hline $\mathrm{Ba}$ & $\mathrm{Ba}$ & $\mathrm{AB}$ Dor Ba & $14.9^{*}$ & {$[12.70]$} & $\ldots$ & M4 & 3150 & $\ldots$ & {$[0.013]$} & {$[0.24]$} & Q 9.'2 & $2 \mathrm{M}$ \\
\hline $\mathrm{Bb}$ & $\mathrm{Bb}$ & $\mathrm{AB}$ Dor $\mathrm{Bb}$ & $14.9^{*}$ & $\ldots$ & $\ldots$ & $\ldots$ & $\ldots$ & $\ldots$ & $\ldots$ & $\ldots$ & Q $0^{\prime \prime} 06$ & T08 \\
\hline$\ldots$ & $\ldots$ & UX Col & 57 & 10.589 & 7.76 & $\mathrm{~K} 3$ & 4730 & $\ldots$ & 0.23 & 0.12 & $\ldots$ & $\ldots$ \\
\hline$\ldots$ & $\ldots$ & Parenago 2752 & 116 & 10.830 & 9.14 & G8 & 5570 & $\ldots$ & 0.60 & 0.07 & $\ldots$ & $\ldots$ \\
\hline$\ldots$ & $\ldots$ & CPD-19 878 & 71 & 10.710 & 8.12 & K1 & 5080 & $\ldots$ & 0.28 & 0.09 & $\ldots$ & $\ldots$ \\
\hline$\ldots$ & $\ldots$ & TYC 7605-1429-1 & 128 & 12.281 & 9.12 & K4 & 4590 & $\ldots$ & 0.25 & 0.06 & $\ldots$ & $\ldots$ \\
\hline$\ldots$ & $\ldots$ & CD-26 2425 & 70 & 10.870 & 8.47 & $\mathrm{~K} 2$ & 4900 & $\ldots$ & 0.25 & 0.09 & $\ldots$ & $\ldots$ \\
\hline 39576 & 27727 & $\mathrm{TZ} \mathrm{Col}$ & $87.6 \mathrm{H}$ & 9.034 & 7.52 & G3 & 5820 & $\ldots$ & 1.7 & 0.14 & $\ldots$ & $\ldots$ \\
\hline$\ldots$ & $\ldots$ & TY Col & 68 & 9.527 & 7.63 & G6 & 5690 & $\ldots$ & 0.66 & 0.11 & $\ldots$ & $\ldots$ \\
\hline$\cdots$ & $\cdots$ & BD-13 1328 & 39 & 10.587 & 7.77 & K4 & 4590 & $\cdots$ & 0.11 & 0.13 & $\cdots$ & $\ldots$ \\
\hline$\cdots$ & $\ldots$ & CD-34 2676 & 72 & 10.160 & 8.20 & G9 & 5410 & $\ldots$ & 0.44 & 0.10 & $\ldots$ & $\ldots$ \\
\hline$\ldots$ & $\ldots$ & $\mathrm{CD}-352722 \mathrm{~A}$ & 24 & 10.954 & {$[7.05]$} & M1 & 3650 & $\ldots$ & 0.074 & 0.26 & B & $\ldots$ \\
\hline$\ldots$ & $\ldots$ & CD-35 2722 B & 24 & $\ldots$ & $\ldots$ & $\mathrm{L} 4$ & $\ldots$ & $\ldots$ & $\ldots$ & $\ldots$ & В $0 ! 07$ & W11 \\
\hline 45270 & 30314 & CD-60 1425 & $23.5 \mathrm{H}$ & 6.517 & {$[5.05]$} & G1 & 5940 & $\ldots$ & 1.3 & 0.41 & $\mathrm{~S}$ & $\mathrm{NC} 10$ \\
\hline$\ldots$ & $\ldots$ & GSC 8894-0426 & 24 & 12.7 & 7.21 & M3 & 3350 & $\ldots$ & 0.027 & 0.19 & $\ldots$ & $\ldots$ \\
\hline
\end{tabular}


Table 1-Continued

\begin{tabular}{|c|c|c|c|c|c|c|c|c|c|c|c|c|}
\hline $\mathrm{HD}$ & HIP & Other & $\begin{array}{c}\operatorname{Dist}^{a} \\
(\mathrm{pc})\end{array}$ & $\begin{array}{c}V^{b} \\
\mathrm{mag}\end{array}$ & $\begin{array}{c}K^{b} \\
\mathrm{mag}\end{array}$ & $\begin{array}{l}\text { Prev. }^{c} \\
\text { SpT }\end{array}$ & $\begin{array}{l}T_{\text {eff }} \\
(\mathrm{K})\end{array}$ & $\begin{array}{c}\text { New } \\
\text { SpT, } T_{\text {eff }}\end{array}$ & $\begin{array}{l}\operatorname{Lum}^{b} \\
\left(L_{\odot}\right)\end{array}$ & $\begin{array}{c}\theta^{b} \\
(\mathrm{mas})\end{array}$ & $\begin{array}{c}\text { Known }^{e} \\
\text { Mult }\end{array}$ & $\begin{array}{c}\text { Mult }^{f} \\
\text { Ref }\end{array}$ \\
\hline$\ldots$ & $\ldots$ & GSC 8544-1037 & 143 & 11.5 & 8.93 & K4 & 4590 & $\cdots$ & 0.65 & 0.08 & $\ldots$ & $\cdots$ \\
\hline$\ldots$ & $\ldots$ & CD-57 1654 & 103 & 10.438 & 8.93 & G2 & 5860 & $\ldots$ & 0.63 & 0.07 & $\ldots$ & $\cdots$ \\
\hline$\ldots$ & $\ldots$ & $\mathrm{BD}+201790$ & 26 & 10.022 & 6.88 & K5 & 4400 & $\ldots$ & 0.10 & 0.19 & $\mathrm{~S}$ & E11 \\
\hline 59169 A & 36108 & CD-49 $2843 \mathrm{~A}$ & $118 \mathrm{H}$ & 10.215 & {$[7.93]$} & G7 & 5620 & $\cdots$ & 1.1 & 0.09 & B & $\cdots$ \\
\hline B & B & CD-49 2843 B & $118^{*}$ & 11.148 & $\ldots$ & K3: & 4700 & $\cdots$ & 0.59 & 0.09 & B $11^{\prime \prime} 2$ & T08 \\
\hline$\ldots$ & 36349 A & V372 Pup A & $15.6 \mathrm{H}$ & {$[10.077]$} & {$[5.72]$} & M1 & 3650 & $\cdots$ & {$[0.073]$} & [0.39] & $\mathrm{T}$ & $\cdots$ \\
\hline$\ldots$ & $\mathrm{B}$ & V372 Pup B & $15.6^{*}$ & $\ldots$ & $\ldots$ & $\cdots$ & $\cdots$ & $\cdots$ & $\ldots$ & $\ldots$ & $\mathrm{T} 0^{\prime \prime} 2$ & T08 \\
\hline$\ldots$ & $\mathrm{C}$ & V372 Pup C & $15.6^{*}$ & $\ldots$ & $\ldots$ & $\ldots$ & $\ldots$ & $\ldots$ & $\ldots$ & $\ldots$ & $\mathrm{T} 6 .{ }^{\prime \prime} 6$ & T08 \\
\hline$\ldots$ & $\ldots$ & CD-84 80 & 71 & 9.961 & 7.91 & G9 & 5410 & $\ldots$ & 0.51 & 0.11 & $\ldots$ & $\ldots$ \\
\hline $64982 \mathrm{~A}$ & $37855 \mathrm{~A}$ & CD-79 $300 \mathrm{~A}$ & $83.5 \mathrm{H}$ & 8.955 & {$[7.59]$} & G0 & 6030 & $\cdots$ & 1.6 & 0.13 & B & $\cdots$ \\
\hline B & B & CD-79 300 B & $83.5^{*}$ & $\ldots$ & 8.88 & $\cdots$ & $\ldots$ & $\ldots$ & $\ldots$ & $\ldots$ & B $5 . \prime 7$ & $2 \mathrm{M}$ \\
\hline$\cdots$ & $\ldots$ & BD-07 2388 & 93 & 9.373 & 6.92 & K1 & 5080 & $\cdots$ & 1.7 & 0.17 & $\ldots$ & $\cdots$ \\
\hline 82879 & $\ldots$ & RX J0928.5-7815 & $58 \mathrm{c}$ & 8.987 & 7.83 & $\mathrm{~F} 4$ & 6580 & $\cdots$ & 0.74 & 0.11 & $\ldots$ & $\cdots$ \\
\hline$\ldots$ & $\ldots$ & CD-45 5772 & 70 & 10.888 & 8.07 & K4 & 4590 & $\ldots$ & 0.19 & 0.09 & $\ldots$ & $\ldots$ \\
\hline$\cdots$ & 51317 & GJ 393 & $7.23 \mathrm{H}$ & 9.586 & 5.31 & M2 & 3500 & $\mathrm{M} 2.5,3420$ & 0.035 & 0.69 & $\mathrm{~S}$ & L07,NC10,E11 \\
\hline $99827 \mathrm{~A}$ & $55746 \mathrm{~A}$ & CD-84 $114 \mathrm{~A}$ & $82.9 \mathrm{H}$ & {$[7.638]$} & {$[6.49]$} & F5 & 6440 & $\ldots$ & {$[5.2]$} & {$[0.21]$} & B & $\cdots$ \\
\hline B & B & CD-84 114 B & $82.9^{*}$ & $\ldots$ & $\ldots$ & $\ldots$ & $\ldots$ & $\ldots$ & $\ldots$ & $\ldots$ & В $33^{\prime \prime} 5$ & T08 \\
\hline$\cdots$ & $\ldots$ & TYC 4943-192-1 & 45.5 & 11.344 & 7.83 & M0 & 3800 & $\ldots$ & 0.16 & 0.19 & $\ldots$ & $\cdots$ \\
\hline $113449 \mathrm{~A}$ & $63742 \mathrm{~A}$ & PX Vir A & $22.1 \mathrm{H}$ & {$[7.700]$} & {$[5.51]$} & K1 & 5080 & $\mathrm{~K} 1,5020$ & {$[0.43]$} & {$[0.36]$} & B & \\
\hline $\mathrm{B}$ & $\mathrm{B}$ & PX Vir B & $22.1^{*}$ & $\ldots$ & $\ldots$ & $\ldots$ & $\ldots$ & $\ldots$ & $\ldots$ & $\ldots$ & $0 ! .4$ & T08,NC10,E11 \\
\hline $139751 \mathrm{~A}$ & 76768 & BD-18 $4125 \mathrm{~A}$ & $42.6 \mathrm{H}$ & {$[10.377]$} & {$[6.95]$} & K5 & 4400 & $\ldots$ & {$[0.19]$} & {$[0.16]$} & B & $\ldots$ \\
\hline B & $\ldots$ & BD-18 $4125 \mathrm{~B}$ & $42.6^{*}$ & $\ldots$ & $\ldots$ & $\ldots$ & $\ldots$ & $\ldots$ & $\ldots$ & $\ldots$ & B $0 . \prime 9$ & T08 \\
\hline$\cdots$ & 81084 & LP 745-70 & $31.9 \mathrm{H}$ & 11.178 & 7.55 & M0 & 3800 & $\cdots$ & 0.090 & 0.20 & $\mathrm{~S}$ & L07,NC10,E11 \\
\hline 152555 & 82688 & BD-04 4194 & $47.6 \mathrm{H}$ & 7.818 & 6.36 & G0 & 6030 & G0,6000 & 1.5 & 0.22 & S & E11 \\
\hline 317617 & $\cdots$ & TYC 7379-279-1 & 56 & 10.452 & 7.67 & K3 & 4730 & $\cdots$ & 0.25 & 0.12 & $\ldots$ & $\cdots$ \\
\hline 159911 & $\cdots$ & BD-13 4687 & 45 & 10.100 & 6.84 & K4 & 4590 & $\cdots$ & 0.23 & 0.16 & $\ldots$ & $\cdots$ \\
\hline $160934 \mathrm{~A}$ & 86346 & GJ $4020 \mathrm{~A}$ & $24.5 \mathrm{H}$ & {$[10.150]$} & {$[6.81]$} & K7 & 4000 & $\mathrm{~K} 7.5,3900$ & {$[0.12]$} & {$[0.29]$} & $\mathrm{T}$ & NC10,E11 \\
\hline B & $\ldots$ & GJ $4020 \mathrm{~B}$ & $24.5^{*}$ & $\ldots$ & $\ldots$ & $\cdots$ & $\ldots$ & $\ldots$ & $\ldots$ & $\ldots$ & $\mathrm{T} 0 . \prime 2$ & T08,NC10,E11 \\
\hline $\mathrm{C}$ & $\ldots$ & GJ $4020 \mathrm{C}$ & $24.5^{*}$ & $\ldots$ & 9.42 & $\ldots$ & $\ldots$ & $\ldots$ & $\ldots$ & $\cdots$ & Т $199^{\prime \prime} 1$ & $2 \mathrm{M}$ \\
\hline $176367 \mathrm{~A}$ & $93375 \mathrm{~A}$ & CD-28 15269 A & $62.8 \mathrm{H}$ & 8.469 & 7.15 & G1 & 5940 & $\ldots$ & 1.4 & 0.17 & B & $\cdots$ \\
\hline B & B & CD-28 $15269 \mathrm{~B}$ & $62.8^{*}$ & $\ldots$ & 12.72 & $\ldots$ & $\ldots$ & $\ldots$ & $\ldots$ & $\ldots$ & B $11^{\prime \prime} 2$ & $2 \mathrm{M}$ \\
\hline 177178 & 93580 & $\mathrm{BD}+013865$ & $55.0 \mathrm{H}$ & 5.815 & 5.32 & A4 & 8460 & $\ldots$ & 14 & 0.30 & $\ldots$ & $\cdots$ \\
\hline 178085 & 94235 & CD-60 7126 & $57.2 \mathrm{H}$ & 8.308 & 6.88 & G1 & 5940 & $\ldots$ & 1.4 & 0.18 & $\ldots$ & $\ldots$ \\
\hline 181869 & 95347 & $\alpha \operatorname{Sgr}$ & $52.1 \mathrm{H}$ & 3.943 & 4.20 & B8 & 11900 & $\cdots$ & 130 & 0.48 & $\ldots$ & $\cdots$ \\
\hline
\end{tabular}


Table 1 - Continued

\begin{tabular}{|c|c|c|c|c|c|c|c|c|c|c|c|c|}
\hline HD & HIP & Other & $\begin{array}{c}\text { Dist }^{a} \\
(\mathrm{pc})\end{array}$ & $\begin{array}{c}V^{b} \\
\mathrm{mag}\end{array}$ & $\begin{array}{c}K^{b} \\
\mathrm{mag}\end{array}$ & $\begin{array}{c}\text { Prev. }^{c} \\
\text { SpT }\end{array}$ & $\begin{array}{l}T_{\text {eff }} \\
(\mathrm{K})\end{array}$ & $\begin{array}{c}\text { New } \\
\text { SpT, } T_{\text {eff }}\end{array}$ & $\begin{array}{c}\operatorname{Lum}^{b} \\
\left(L_{\odot}\right)\end{array}$ & $\begin{array}{c}\theta^{b} \\
(\mathrm{mas}) \\
\end{array}$ & $\begin{array}{c}\text { Known }^{e} \\
\text { Mult }\end{array}$ & $\begin{array}{c}\text { Mult }^{f} \\
\text { Ref }\end{array}$ \\
\hline$\cdots$ & $\ldots$ & TYC 486-4943-1 & 71 & 11.283 & 8.66 & K3 & 4730 & $\cdots$ & 0.19 & 0.08 & $\ldots$ & $\ldots$ \\
\hline 189285 & $\cdots$ & BD-04 4987 & 95 & 9.433 & 7.84 & G7 & 5620 & $\cdots$ & 1.4 & 0.12 & $\cdots$ & $\cdots$ \\
\hline$\cdots$ & $\ldots$ & BD-03 4778 & 70 & 10.011 & 7.92 & K1 & 5080 & $\cdots$ & 0.52 & 0.12 & $\cdots$ & $\cdots$ \\
\hline$\cdots$ & $\ldots$ & $\mathrm{BD}+054576$ & 38.5 & 10.518 & 7.13 & K7 & 4000 & $\ldots$ & 0.19 & 0.22 & $\ldots$ & $\ldots$ \\
\hline 199058 & $\cdots$ & $\mathrm{BD}+084561$ & 75 & 8.614 & 6.97 & G5 & 5750 & G6,5710 & 1.9 & 0.17 & $\cdots$ & $\cdots$ \\
\hline$\cdots$ & $\cdots$ & TYC 1090-543-1 & 75 & 11.284 & 8.82 & K4 & 4590 & $\cdots$ & 0.22 & 0.09 & $\cdots$ & $\cdots$ \\
\hline 201919 & $\cdots$ & GSC 06351-00286 & 39 & 10.433 & 7.58 & K6 & 4260 & $\cdots$ & 0.18 & 0.19 & $\cdots$ & $\cdots$ \\
\hline$\cdots$ & 106231 & LO Peg & $25.1 \mathrm{H}$ & 9.245 & 6.38 & K5 & 4400 & $\mathrm{~K} 5,4450$ & 0.19 & 0.27 & $\mathrm{~S}$ & NC10,E11 \\
\hline 207278 & 107684 & CD-40 14502 & $83.7 \mathrm{H}$ & 9.614 & 7.98 & G7 & 5620 & $\ldots$ & 0.95 & 0.11 & $\ldots$ & $\ldots$ \\
\hline$\ldots$ & 107948 & GJ 4231 & $31.8 \mathrm{H}$ & 12.040 & 7.39 & M2 & 3500 & $\cdots$ & 0.059 & 0.19 & $\ldots$ & $\cdots$ \\
\hline 209952 & 109268 & $\alpha$ Gru & $31.1 \mathrm{H}$ & 1.7 & 2.02 & B6 & 14000 & $\cdots$ & 520 & 1.17 & $\cdots$ & $\cdots$ \\
\hline$\ldots$ & $110526 \mathrm{~A}$ & GJ $856 \mathrm{~A}$ & $15.5 \mathrm{H}$ & 11.420 & {$[6.05]$} & M3 & 3350 & $\cdots$ & 0.038 & 0.34 & $\mathrm{~B}$ & E11 \\
\hline$\cdots$ & B & GJ 856 B & $15.5^{*}$ & 11.830 & $\cdots$ & M3 & 3350 & $\cdots$ & 0.026 & 0.28 & B $1 . ! 8$ & T08 \\
\hline 217343 & 113579 & CD-26 16415 & $32.0 \mathrm{H}$ & 7.484 & 5.94 & G5 & 5750 & $\cdots$ & 0.95 & 0.29 & S & E11 \\
\hline 217379 A & $113597 \mathrm{~A}$ & CD-26 16420 A & $30.0 \mathrm{H}$ & {$[10.024]$} & {$[6.27]$} & $\mathrm{K} 7$ & 4000 & $\ldots$ & {$[0.19]$} & {$[0.28]$} & $\mathrm{T}$ & $\ldots$ \\
\hline $\mathrm{B}$ & B & CD-26 16420 B & $30.0^{*}$ & {$[10.620]$} & $\cdots$ & $\cdots$ & $\cdots$ & $\cdots$ & $\cdots$ & $\cdots$ & $\mathrm{T} 1 . ! 8$ & T08 \\
\hline $\mathrm{C}$ & $\mathrm{C}$ & CD-26 $16420 \mathrm{C}$ & $30.0^{*}$ & $\ldots$ & $\cdots$ & $\cdots$ & $\ldots$ & $\cdots$ & $\ldots$ & $\ldots$ & $\mathrm{T} \mathrm{SB}$ & T08 \\
\hline$\cdots$ & 114066 & GJ 9809 & $24.9 \mathrm{H}$ & 10.922 & 6.98 & M1 & 3650 & M0.5,3720 & 0.076 & 0.25 & $\mathrm{~S}$ & NC10,E11 \\
\hline $218860 \mathrm{~A}$ & 114530 & CD-45 14955 A & $50.6 \mathrm{H}$ & 8.803 & 7.03 & G8 & 5570 & $\cdots$ & 0.75 & 0.17 & B & $\cdots$ \\
\hline $\mathrm{B}$ & B & CD-45 14955 B & $50.6^{*}$ & 13.0 & 8.85 & M3 & 3350 & $\ldots$ & 0.091 & 0.16 & В $199^{\prime \prime} 6$ & $2 \mathrm{M}$ \\
\hline$\ldots$ & 115162 & $\mathrm{BD}+414749$ & $49.4 \mathrm{H}$ & 8.929 & 7.22 & G4 & 5780 & $\ldots$ & 0.58 & 0.14 & S & E11 \\
\hline 220825 & 115738 & $\kappa \mathrm{Psc}_{\mathrm{sc}}$ & $49.7 \mathrm{H}$ & 4.917 & 4.90 & A0 & 9520 & $\ldots$ & 30 & 0.38 & $\ldots$ & $\ldots$ \\
\hline 222575 & 116910 & CD-36 15990 & $62.3 \mathrm{H}$ & 9.382 & 7.62 & G8 & 5570 & $\cdots$ & 0.65 & 0.13 & $\cdots$ & $\cdots$ \\
\hline 223352 & 117452 & $\delta \mathrm{Scl}$ & $44 \mathrm{H}$ & 4.6 & 4.53 & A0 & 9520 & $\cdots$ & 32 & 0.44 & $\cdots$ & $\cdots$ \\
\hline 224228 & 118008 & GJ 4377 & $22.1 \mathrm{H}$ & 8.248 & 5.91 & $\mathrm{~K} 2$ & 4900 & $\cdots$ & 0.30 & 0.31 & $\mathrm{~S}$ & NC10,E11 \\
\hline
\end{tabular}

Note. - ${ }^{a}$ Distances marked with an $\mathrm{H}$ are from Hipparcos parallax measurements, those marked with an asterisk are assumed to be that of their companion, and those marked with a c are calculated based on an assumed radius (see Section 2).

${ }^{b}$ Values in brackets indicate measurements that may be biased by a spatially unresolved companion.

${ }^{c}$ Spectral types followed by a colon are estimated from B-V color (see Section 2)

e Multiplicity abbreviations: S is Single, B is Binary, T is Triple, and Q is Quadruple

$f$ Separation References: $2 \mathrm{M}=2 \mathrm{MASS}$; B07 = Biller et al. (2007); B10 = Biller et al. (2010); E11 = Evans et al. (2011); K07 = Kasper et al. (2007); L07 = Lafreniere et al. (2007); M90 = McAlister et al. (1990); NC10 = Nielson \& Close 2010; T08 = Torres et al. (2008); W11 = Wahhaj et al. (2011) 
Table 2. Proposed $\beta$ Pictoris Moving Group Members

\begin{tabular}{|c|c|c|c|c|c|c|c|c|c|c|c|c|}
\hline HD & HIP & Other & $\begin{array}{c}\operatorname{Dist}^{a} \\
(\mathrm{pc})\end{array}$ & $\begin{array}{c}V^{b} \\
\mathrm{mag}\end{array}$ & $\begin{array}{c}K^{b} \\
\mathrm{mag}\end{array}$ & $\begin{array}{c}\text { Prev. }^{c} \\
\text { SpT }\end{array}$ & $\begin{array}{l}T_{\text {eff }} \\
(\mathrm{K})\end{array}$ & $\begin{array}{c}\text { New } \\
\text { SpT, } T_{\text {eff }}\end{array}$ & $\begin{array}{l}\operatorname{Lum}^{b} \\
\left(L_{\odot}\right)\end{array}$ & $\begin{array}{c}\theta^{b} \\
(\mathrm{mas})\end{array}$ & $\begin{array}{c}\text { Known }^{e} \\
\text { Mult }\end{array}$ & $\begin{array}{c}\text { Mult }^{f} \\
\text { Ref }\end{array}$ \\
\hline 203 & 560 & HR9 & $39.1 \mathrm{H}$ & 6.173 & 5.24 & F3 & 6730 & $\cdots$ & 4.5 & 0.37 & $\mathrm{~S}$ & E11 \\
\hline$\cdots$ & $\cdots$ & $\mathrm{BD}+17232 \mathrm{~A}$ & 52.6 & 10.584 & {$[6.72]$} & K3 & 4730 & $\cdots$ & 0.20 & 0.12 & B & $\cdots$ \\
\hline$\cdots$ & $\ldots$ & $\mathrm{BD}+17232 \mathrm{~B}$ & $52.6^{*}$ & 10.643 & $\cdots$ & K5: & 4420 & $\ldots$ & 0.23 & 0.14 & В $1 . \prime 8$ & M00 \\
\hline$\cdots$ & $\cdots$ & TYC 1186-706-1 & 60 & 10.841 & 7.34 & K7.5 & 3940 & $\mathrm{~K} 7,4000$ & 0.35 & 0.19 & $\cdots$ & $\cdots$ \\
\hline$\cdots$ & $\cdots$ & 1RXS J010711.7-193529 & 54 & 11.290 & 7.25 & M1 & 3650 & .. & 0.27 & 0.23 & $\cdots$ & $\cdots$ \\
\hline $14082 \mathrm{~A}$ & 10680 & $\mathrm{BD}+28382 \mathrm{~A}$ & $39.4 \mathrm{H}$ & 7.031 & 5.79 & F5 & 6440 & $\mathrm{~F} 8,6120$ & 2.0 & 0.30 & $\mathrm{~B}$ & E11 \\
\hline $\mathrm{B}$ & 10679 & $\mathrm{BD}+28382 \mathrm{~B}$ & $34.0 \mathrm{H}$ & 7.755 & 6.26 & G2 & 5860 & G1,5970 & 0.81 & 0.23 & B $13 ! ! 8$ & $2 \mathrm{M}$ \\
\hline$\cdots$ & 11152 & LP $353-51$ & $30.7 \mathrm{H}$ & 11.307 & 7.35 & M3 & 3350 & $\cdots$ & 0.16 & 0.36 & $\ldots$ & $\ldots$ \\
\hline 15115 & 11360 & $\mathrm{BD}+05338$ & $44.8 \mathrm{H}$ & 6.781 & 5.82 & $\mathrm{~F} 4$ & 6580 & F8,6190 & 3.42 & 0.33 & $\cdots$ & $\cdots$ \\
\hline$\cdots$ & $11437 \mathrm{~A}$ & AG Tri A & $42.3 \mathrm{H}$ & 10.1 & 7.08 & K6 & 4260 & $\cdots$ & 0.29 & 0.22 & $\mathrm{~B}$ & E11 \\
\hline$\cdots$ & B & AG Tri B & $42.3^{*}$ & 12.4 & 7.92 & M2 & 3500 & $\ldots$ & 0.070 & 0.16 & B $22^{\prime \prime} 1$ & 2M,E11 \\
\hline$\ldots$ & $\ldots$ & TYC 7558-655-1 & 35.7 & 10.417 & 7.23 & K5 & 4395 & $\ldots$ & 0.13 & 0.16 & $\ldots$ & $\ldots$ \\
\hline$\cdots$ & 12545 & $\mathrm{BD}+05378$ & $40.5 \mathrm{H}$ & 10.194 & 7.07 & K6 & 4260 & $\cdots$ & 0.25 & 0.21 & $\mathrm{~S}$ & $\mathrm{~B} 07, \mathrm{~K} 07, \mathrm{NC} 10, \mathrm{E} 11$ \\
\hline 29391 A & 21547 & 51 Eri A & $29.8 \mathrm{H}$ & 5.209 & 4.54 & F0 & 7200 & $\ldots$ & 6.7 & 0.52 & $\mathrm{~T}$ & E11 \\
\hline $\mathrm{Ba}$ & $\mathrm{Ba}$ & GJ $3305 \mathrm{~A}$ & 30 & {$[10.6]$} & {$[6.41]$} & M1 & 3650 & $\cdots$ & {$[0.16]$} & {$[0.31]$} & $\mathrm{T} 66^{\prime \prime} 5$ & $2 \mathrm{M}$ \\
\hline $\mathrm{Bb}$ & $\mathrm{Bb}$ & GJ $3305 \mathrm{~B}$ & 30 & $\cdots$ & $\ldots$ & $\cdots$ & $\ldots$ & $\ldots$ & $\ldots$ & $\ldots$ & T 0.! 09 & K07 \\
\hline$\ldots$ & 23200 & V1005 Ori & $26.7 \mathrm{H}$ & 10.097 & 6.26 & M0 & 3800 & $\ldots$ & 0.17 & 0.33 & $\ldots$ & $\ldots$ \\
\hline$\ldots$ & 23309 & CD-57 1054 & $26.3 \mathrm{H}$ & 10.105 & 6.24 & M0 & 3800 & $\ldots$ & 0.16 & 0.33 & $\mathrm{~S}$ & $\mathrm{~B} 07, \mathrm{NC} 10$ \\
\hline$\ldots$ & 23418 Aa & LP 476-207 Aa & $32.1 \mathrm{H}$ & [11.741] & {$[6.37]$} & M3 & 3350 & $\ldots$ & {$[0.11]$} & {$[0.29]$} & $\mathrm{B}$ & $\ldots$ \\
\hline$\ldots$ & $23418 \mathrm{Ab}$ & $\mathrm{LP} 476-207 \mathrm{Ab}$ & $32.1 *$ & $\ldots$ & $\ldots$ & $\ldots$ & $\ldots$ & $\ldots$ & $\ldots$ & $\ldots$ & B $1{ }^{\prime \prime} 0$ & T08 \\
\hline$\ldots$ & $\ldots$ & BD-21 $1074 \mathrm{~A}$ & 18 & 10.438 & 6.11 & M1 & 3650 & $\ldots$ & 0.056 & 0.31 & $\mathrm{~T}$ & $\ldots$ \\
\hline$\ldots$ & $\ldots$ & B & $18^{*}$ & [11.500] & {$[6.12]$} & $\cdots$ & $\ldots$ & $\ldots$ & $\ldots$ & $\ldots$ & $\mathrm{T} 8 . ! 2$ & $2 \mathrm{M}$ \\
\hline$\ldots$ & $\ldots$ & $\mathrm{C}$ & $18^{*}$ & $\ldots$ & $\ldots$ & M3 & 3350 & $\ldots$ & $\ldots$ & $\ldots$ & $\mathrm{T} 1^{\prime \prime} 2$ & T08 \\
\hline $35850 \mathrm{~A}$ & $25486 \mathrm{~A}$ & AF Lep A & $26.8 \mathrm{H}$ & [6.296] & {$[4.93]$} & F7 & 6270 & $\ldots$ & {$[1.9]$} & {$[0.40]$} & $\mathrm{B}$ & E11 \\
\hline $\mathrm{B}$ & $\mathrm{B}$ & B & $26.8^{*}$ & $\ldots$ & $\ldots$ & $\ldots$ & $\ldots$ & $\ldots$ & $\ldots$ & $\ldots$ & B SB & T08 \\
\hline$\ldots$ & $\ldots$ & V1311 Ori & 36 & 11.098 & 7.01 & M2 & 3500 & $\ldots$ & 0.18 & 0.30 & $\ldots$ & $\ldots$ \\
\hline 39060 & 27321 & $\beta \mathrm{Pic}$ & $19.3 \mathrm{H}$ & 3.848 & 3.53 & A3 & 8720 & $\cdots$ & 10 & 0.69 & $\ldots$ & $\cdots$ \\
\hline 45081 & 29964 & AO Men & $38.5 \mathrm{H}$ & 9.989 & 6.81 & K4 & 4590 & $\ldots$ & 0.20 & 0.17 & $\mathrm{~S}$ & $\mathrm{~B} 07, \mathrm{~K} 07, \mathrm{NC} 10$ \\
\hline $139084 \mathrm{~A}$ & $76629 \mathrm{~A}$ & V343 Nor A & $39.8 \mathrm{H}$ & 8.153 & 5.85 & Ko & 5350 & $\ldots$ & 0.89 & 0.26 & $\mathrm{~B}$ & $\mathrm{NC} 10$ \\
\hline B & B & B & $39.8^{*}$ & 14.8 & 9.19 & M5 & 3000 & $\ldots$ & 0.036 & 0.16 & B $10^{\prime \prime} 4$ & $2 \mathrm{M}$ \\
\hline$\ldots$ & $\ldots$ & J16430128-1754274 & 57 & $\ldots$ & 8.55 & M0.5 & 3700 & $\ldots$ & $\ldots$ & $\ldots$ & $\ldots$ & $\ldots$ \\
\hline $155555 \mathrm{~A}$ & $84586 \mathrm{~A}$ & V824 Ara A & $31.4 \mathrm{H}$ & [6.869] & {$[4.70]$} & G7 & 5620 & $\cdots$ & {$[1.6]$} & {$[0.40]$} & $\mathrm{T}$ & $\mathrm{B} 07, \mathrm{NC} 10$ \\
\hline B & B & V824 Ara B & $31.4^{*}$ & $\ldots$ & $\ldots$ & $\cdots$ & $\ldots$ & $\cdots$ & $\ldots$ & $\ldots$ & $\mathrm{T} \mathrm{SB}$ & $\mathrm{B} 07, \mathrm{~T} 08, \mathrm{NC} 10$ \\
\hline $\mathrm{C}$ & $\mathrm{C}$ & V824 Ara C & $31.4^{*}$ & {$[12.8]$} & {$[7.63]$} & M3 & 3350 & $\ldots$ & {$[0.040]$} & {$[0.18]$} & T $34^{\prime \prime} 0$ & $2 \mathrm{M}, \mathrm{NC} 10$ \\
\hline
\end{tabular}


Table 2-Continued

\begin{tabular}{|c|c|c|c|c|c|c|c|c|c|c|c|c|}
\hline $\mathrm{HD}$ & HIP & Other & $\begin{array}{c}\text { Dist }^{a} \\
(\mathrm{pc})\end{array}$ & $\begin{array}{c}V^{b} \\
\mathrm{mag}\end{array}$ & $\begin{array}{c}K^{b} \\
\mathrm{mag}\end{array}$ & $\begin{array}{c}\text { Prev. }^{c} \\
\text { SpT }\end{array}$ & $\begin{array}{l}T_{\text {eff }} \\
(\mathrm{K})\end{array}$ & $\begin{array}{c}\text { New } \\
\text { SpT, } T_{\text {eff }}\end{array}$ & $\begin{array}{l}\operatorname{Lum}^{b} \\
\left(L_{\odot}\right)\end{array}$ & $\begin{array}{c}\theta^{b} \\
(\mathrm{mas})\end{array}$ & $\begin{array}{c}\text { Known }^{e} \\
\text { Mult }\end{array}$ & $\begin{array}{c}\text { Mult }^{f} \\
\text { Ref }\end{array}$ \\
\hline$\cdots$ & $\cdots$ & GSC $8350-1924 \mathrm{~A}$ & 76 & {$[13.47]$} & [7.99] & M3 & 3350 & $\ldots$ & {$[0.13]$} & {$[0.13]$} & $\mathrm{B}$ & $\ldots$ \\
\hline$\cdots$ & $\ldots$ & GSC $8350-1924$ B & $76^{*}$ & $\ldots$ & $\ldots$ & $\cdots$ & $\ldots$ & $\ldots$ & $\ldots$ & $\ldots$ & B $0 . \prime 8$ & T08 \\
\hline$\ldots$ & $\ldots$ & CD-54 7336 & 66 & 9.529 & 7.36 & K1 & 5080 & $\ldots$ & 0.72 & 0.16 & $\cdots$ & $\ldots$ \\
\hline 160305 & 86598 & CD-50 11467 & $76 \mathrm{H}$ & 8.357 & 6.99 & F9 & 6110 & $\cdots$ & 2.3 & 0.17 & $\ldots$ & $\cdots$ \\
\hline $161460 \mathrm{~A}$ & $\ldots$ & CD-15 7414 A & 74 & {$[8.986]$} & {$[6.78]$} & K0 & 5250 & $\ldots$ & {$[1.4]$} & {$[0.18]$} & B & $\cdots$ \\
\hline $\mathrm{B}$ & $\cdots$ & CD-15 7414 B & $74^{*}$ & $\cdots$ & $\cdots$ & $\cdots$ & $\cdots$ & $\cdots$ & $\ldots$ & $\cdots$ & B $0 . ! 1$ & T08 \\
\hline $164249 \mathrm{~A}$ & 88399 A & CD-51 11312 A & $46.9 \mathrm{H}$ & 7.007 & 5.91 & F6 & 6350 & $\cdots$ & 3.0 & 0.28 & $\mathrm{~B}$ & $\cdots$ \\
\hline B & 88399 B & CD-51 11312 B & $46.9^{*}$ & 12.5 & 8.27 & M2 & 3500 & $\ldots$ & 0.083 & 0.16 & B $6 . " 4$ & $2 \mathrm{M}$ \\
\hline $165189 \mathrm{~A}$ & $\ldots$ & HR6749 A & 44 & 5.624 & {$[6.39]$} & A5 & 8200 & $\ldots$ & 11 & 0.34 & $\mathrm{~B}$ & $\ldots$ \\
\hline B & $\ldots$ & B & $44^{*}$ & 5.650 & $\ldots$ & A8: & 7550 & $\ldots$ & 10 & 0.39 & B $1 . \prime 7$ & T08 \\
\hline 319139 A & $\cdots$ & V4046 Sgr A & 73 & [10.681] & {$[7.25]$} & K6 & 4260 & $\cdots$ & {$[0.51]$} & {$[0.17]$} & $\mathrm{B}$ & $\cdots$ \\
\hline B & $\ldots$ & V4046 Sgr B & $73^{*}$ & $\ldots$ & $\ldots$ & $\ldots$ & $\ldots$ & $\ldots$ & $\ldots$ & $\ldots$ & B SB & T08 \\
\hline$\ldots$ & $\ldots$ & GSC 7396-0759 & 73 & 12.8 & 8.54 & M1 & 3650 & $\ldots$ & 0.12 & 0.11 & $\ldots$ & $\ldots$ \\
\hline 168210 & 89829 & CD-29 14813 & $75.5 \mathrm{H}$ & 8.797 & 7.05 & G5 & 5750 & $\ldots$ & 1.6 & 0.16 & $\ldots$ & $\ldots$ \\
\hline $172555 \mathrm{~A}$ & $92024 \mathrm{~A}$ & HR 7012 & $29.2 \mathrm{H}$ & 4.767 & 4.30 & A6 & 8350 & $\ldots$ & 10 & 0.49 & $\mathrm{~T}$ & $\mathrm{~B} 07, \mathrm{NC} 10$ \\
\hline $\mathrm{B}$ & $\mathrm{B}$ & CD-64 $1208 \mathrm{~A}$ & $29^{*}$ & {$[9.5]$} & {$[6.10]$} & K5 & 4400 & $\ldots$ & {$[0.20]$} & {$[0.25]$} & $\mathrm{T} 74^{\prime \prime} 0$ & 2M,B07,NC10 \\
\hline $\mathrm{C}$ & $\mathrm{C}$ & CD-64 1208 B & $29 *$ & $\ldots$ & $\ldots$ & $\ldots$ & $\ldots$ & $\ldots$ & $\ldots$ & $\ldots$ & $\mathrm{T} 0^{\prime \prime} 2$ & Т08 \\
\hline$\ldots$ & $\ldots$ & TYC 9073-0762-1 & 54 & 12.216 & 7.85 & M1 & 3650 & $\ldots$ & 0.12 & 0.15 & $\ldots$ & $\ldots$ \\
\hline$\ldots$ & $\ldots$ & CD-31 16041 & 51 & 11.301 & 7.46 & K7 & 4000 & $\ldots$ & 0.17 & 0.15 & $\ldots$ & $\ldots$ \\
\hline $174429 \mathrm{~A}$ & $92680 \mathrm{~A}$ & PZ Tel A & $49.7 \mathrm{H}$ & 8.406 & 6.37 & G9 & 5410 & $\ldots$ & 1.1 & 0.22 & $\mathrm{~B}$ & $\mathrm{NC} 10$ \\
\hline B & B & PZ Tel B & $49.7^{*}$ & $\ldots$ & $\ldots$ & $\ldots$ & $\ldots$ & $\ldots$ & $\ldots$ & $\ldots$ & B $0 . .3$ & $\mathrm{~B} 10, \mathrm{NC} 10$ \\
\hline$\ldots$ & $\ldots$ & TYC 6872-1011-1 & 79 & 11.897 & 8.02 & M0 & 3800 & $\ldots$ & 0.28 & 0.14 & $\ldots$ & $\ldots$ \\
\hline$\cdots$ & $\cdots$ & CD-26 13904 A & 80 & [10.203] & {$[7.37]$} & $\mathrm{K} 4$ & 4590 & $\cdots$ & {$[0.67]$} & {$[0.15]$} & $\mathrm{B}$ & $\cdots$ \\
\hline$\ldots$ & $\ldots$ & CD-26 13904 B & $80^{*}$ & $\ldots$ & $\ldots$ & $\ldots$ & $\ldots$ & $\ldots$ & $\ldots$ & $\ldots$ & B $1^{\prime \prime} 1$ & T08 \\
\hline $181296 \mathrm{~A}$ & $95261 \mathrm{~A}$ & $\eta$ Tel A & $47.7 \mathrm{H}$ & 5.015 & 5.01 & A0 & 9520 & $\ldots$ & 26 & 0.36 & $\mathrm{~B}$ & $\ldots$ \\
\hline B & B & $\eta$ Tel B & $47.7^{*}$ & $\ldots$ & $\ldots$ & $\ldots$ & $\ldots$ & $\ldots$ & $\ldots$ & $\ldots$ & B $4 . .2$ & T08 \\
\hline 181327 & 95270 & $\eta \mathrm{Tel} \mathrm{C}$ & $50.6 \mathrm{H}$ & 7.037 & 5.91 & F6 & 6350 & $\ldots$ & 3.4 & 0.28 & $\ldots$ & $\ldots$ \\
\hline$\ldots$ & $\ldots$ & TYC 7443-1102-1 & 58 & 11.797 & 7.85 & M0.0 & 3800 & $\mathrm{~K} 7.5,3900$ & 0.15 & 0.14 & $\mathrm{~B}$ & $\ldots$ \\
\hline$\cdots$ & $\cdots$ & 1RXS J195602.8-320720 & 58 & 13.3 & 8.11 & M4 & 3150 & M3.5,3250 & 0.11 & 0.17 & B $26^{\prime \prime} 3$ & $2 \mathrm{M}$ \\
\hline$\ldots$ & $\ldots$ & 1RXS J200136.9-331307 & 62 & 12.3 & 8.24 & M1 & 3650 & $\ldots$ & 0.14 & 0.14 & $\ldots$ & $\ldots$ \\
\hline 191089 & 99273 & CD-26 14819 & $53.5 \mathrm{H}$ & 7.181 & 6.08 & F5 & 6440 & $\ldots$ & 3.2 & 0.25 & $\ldots$ & $\ldots$ \\
\hline $196982 \mathrm{~A}$ & $102141 \mathrm{~A}$ & AT Mic A & $10.2 \mathrm{H}$ & 11.0 & {$[4.94]$} & M4 & 3150 & $\ldots$ & 0.036 & 0.60 & $\mathrm{~B}$ & $\mathrm{~B} 07, \mathrm{NC} 10$ \\
\hline B & B & AT Mic B & $10.2^{*}$ & 11.1 & $\ldots$ & M4 & 3150 & $\ldots$ & 0.033 & 0.57 & B $3 . \prime 3$ & $\mathrm{~B} 07, \mathrm{~T} 08, \mathrm{NC} 10$ \\
\hline 197481 & 102409 & AU Mic & $9.94 \mathrm{H}$ & 8.757 & 4.53 & M1 & 3650 & $\ldots$ & 0.10 & 0.72 & $\mathrm{~S}$ & $\mathrm{~B} 07, \mathrm{E} 11$ \\
\hline
\end{tabular}


Table 2-Continued

\begin{tabular}{|c|c|c|c|c|c|c|c|c|c|c|c|c|}
\hline $\mathrm{HD}$ & HIP & Other & $\begin{array}{c}\text { Dist }^{a} \\
(\mathrm{pc})\end{array}$ & $\begin{array}{c}V^{b} \\
\mathrm{mag}\end{array}$ & $\begin{array}{c}K^{b} \\
\mathrm{mag}\end{array}$ & $\begin{array}{l}\text { Prev. }^{c} \\
\text { SpT }\end{array}$ & $\begin{array}{l}T_{\text {eff }} \\
(\mathrm{K})\end{array}$ & $\begin{array}{c}\text { New } \\
\mathrm{SpT}, T_{\text {eff }}\end{array}$ & $\begin{array}{l}\operatorname{Lum}^{b} \\
\left(L_{\odot}\right)\end{array}$ & $\begin{array}{c}\theta^{b} \\
(\mathrm{mas})\end{array}$ & $\begin{array}{c}\text { Known }^{e} \\
\text { Mult }\end{array}$ & $\begin{array}{c}\text { Mult }^{f} \\
\text { Ref }\end{array}$ \\
\hline 199143 A & $103311 \mathrm{~A}$ & BD-17 $6127 \mathrm{~A}$ & $47.7 \mathrm{H}$ & {$[7.318]$} & {$[5.81]$} & F7 & 6270 & $\ldots$ & {$[2.4]$} & {$[0.25]$} & $\mathrm{B}$ & $\mathrm{NC} 10$ \\
\hline B & B & $\mathrm{B}$ & $47.7^{*}$ & $\ldots$ & $\ldots$ & $\ldots$ & $\ldots$ & $\ldots$ & $\ldots$ & $\ldots$ & B $1 . \prime 1$ & $\mathrm{~T} 08, \mathrm{NC} 10$ \\
\hline $358623 \mathrm{~A}$ & $\ldots$ & AZ Cap A & 47 & {$[10.625]$} & {$[7.04]$} & K6 & 4260 & $\ldots$ & {$[0.22]$} & {$[0.17]$} & $\mathrm{B}$ & E11 \\
\hline B & $\ldots$ & AZ Cap B & $47^{*}$ & $\ldots$ & $\ldots$ & $\cdots$ & $\ldots$ & $\ldots$ & $\ldots$ & $\ldots$ & B $2 . \prime 2$ & T08 \\
\hline$\ldots$ & $\ldots$ & TYC 2211-1309-1 & 46 & 11.367 & 7.72 & M0 & 3800 & $\mathrm{~K} 7,4000$ & 0.13 & 0.15 & $\ldots$ & $\ldots$ \\
\hline$\ldots$ & $\ldots$ & CP-72 2713 & 36 & 10.533 & 6.89 & K7 & 4000 & $\ldots$ & 0.17 & 0.22 & $\ldots$ & $\ldots$ \\
\hline$\ldots$ & $112312 \mathrm{~A}$ & WW PsA & $23.6 \mathrm{H}$ & 12.1 & 6.93 & M4 & 3150 & $\ldots$ & 0.075 & 0.36 & $\mathrm{~B}$ & $\mathrm{~B} 07, \mathrm{NC} 10, \mathrm{E} 11$ \\
\hline$\ldots$ & $\mathrm{B}$ & TX PsA & 20 & 13.4 & 7.79 & M5 & 3000 & $\ldots$ & 0.032 & 0.31 & B $35 !^{\prime \prime} 9$ & 2M,E11 \\
\hline$\ldots$ & $\ldots$ & BD-13 6424 & 28 & 10.685 & 6.57 & M0 & 3800 & $\ldots$ & 0.11 & 0.25 & $\ldots$ & $\ldots$ \\
\hline
\end{tabular}

Note. - ${ }^{a}$ Distances marked with an $\mathrm{H}$ are from Hipparcos parallax measurements, those marked with an asterisk are assumed to be that of their companion, and those marked with a c are calculated based on an assumed radius (see Section 2).

${ }^{b}$ Values in brackets indicate measurements that may be biased by a spatially unresolved companion.

${ }^{c}$ Spectral types followed by a colon are estimated from B-V color (see Section 2)

$f$ Separation References: $2 \mathrm{M}=$ MASS; B07 = Biller et al. (2007); B10 = Biller et al. (2010); E11 = Evans et al. (2011) K07 = Kasper et al. (2007); L07

$=$ Lafreniere et al. (2007); M00 = Morlet et al. (2000); NC10 = Nielson \& Close 2010; T08 = Torres et al. (2008) 
Table 3. $\operatorname{Li}[\mathrm{I}]$ and $\mathrm{H} \alpha$ Equivalent widths

\begin{tabular}{|c|c|c|c|c|}
\hline $\begin{array}{l}\text { Star } \\
\text { Name }\end{array}$ & SNR & $\begin{array}{c}\mathrm{EW}[\mathrm{Li} \mathrm{I}] \\
\AA\end{array}$ & $\begin{array}{c}\mathrm{EW}[\mathrm{H} \alpha] \\
\AA\end{array}$ & MG \\
\hline TYC $1752-63-1^{8}$ & 100 & 0.09 & -1.41 & $\mathrm{AB}$ \\
\hline IS Eri ${ }^{8}$ & 240 & 0.17 & 1.14 & $\mathrm{AB}$ \\
\hline HIP $14809^{8}$ & 275 & 0.14 & 0.94 & $\mathrm{AB}$ \\
\hline HD $25457^{8}$ & 200 & 0.09 & 1.18 & $\mathrm{AB}$ \\
\hline HD $25953^{8}$ & 175 & 0.11 & 1.41 & $\mathrm{AB}$ \\
\hline HIP $51317^{5}$ & 60 & $<0.04$ & 0.38 & $\mathrm{AB}$ \\
\hline PX Vir ${ }^{5}$ & 240 & 0.13 & 0.85 & $\mathrm{AB}$ \\
\hline HD $152555^{5,8}$ & 230 & 0.12 & 1.15 & $\mathrm{AB}$ \\
\hline HD $160934^{8}$ & 125 & $<0.04$ & -0.95 & $\mathrm{AB}$ \\
\hline HD $199058^{8}$ & 150 & 0.16 & 1.08 & $\mathrm{AB}$ \\
\hline LO $\mathrm{Peg}^{8}$ & 350 & 0.15 & -0.6 & $\mathrm{AB}$ \\
\hline HIP $114066^{8}$ & 100 & $<0.04$ & -2 & $\mathrm{AB}$ \\
\hline TYC $1186-706-1^{8}$ & 130 & 0.37 & -0.65 & $\beta$ \\
\hline HIP $10679^{8}$ & 215 & 0.16 & 0.9 & $\beta$ \\
\hline HIP $10680^{8}$ & 175 & 0.11 & 1.59 & $\beta$ \\
\hline HD $15115^{8}$ & 175 & 0.1 & 0.74 & $\beta$ \\
\hline TYC $7443-1102-1^{8}$ & 100 & 0.11 & -0.88 & $\beta$ \\
\hline 1RXS J19506.8-33207208 & 40 & $<0.5$ & -5 & $\beta$ \\
\hline TYC 2211-1309-18 & 150 & $<0.04$ & -1.72 & $\beta$ \\
\hline
\end{tabular}

Note. $-{ }^{5}$ Observed during the May run.

8 Observed during the August run. 